\title{
Towards Secure Over-The-Air Computation
}

\author{
Matthias Frey*, Igor Bjelaković ${ }^{\dagger}$ and Sławomir Stańczak*† \\ ${ }^{*}$ Technische Universität Berlin, Germany and ${ }^{\dagger}$ Fraunhofer Heinrich Hertz Institute, Berlin, Germany
}

\begin{abstract}
We propose a new method to protect Over-The-Air (OTA) computation schemes against passive eavesdropping. Our method uses a friendly jammer whose signal is - contrary to common intuition - stronger at the legitimate receiver than it is at the eavesdropper. It works for a large class of analog OTA computation schemes and we give details on the special case of computing an arithmetic average over an AWGN channel. The derived secrecy guarantee translates to a lower bound on the eavesdropper's mean square error while the question of how to provide operationally more significant guarantees such as semantic security remains open for future work. The key ingredients in proving the security guarantees are a known result on channel resolvability and a generalization of existing achievability results on coding for compound channels.
\end{abstract}

\section{INTRODUCTION}

In many envisioned applications in wireless networks, the receiver requires only a function of values available at the distributed transmitters rather than the full information about the values themselves. Examples include distributed Federated Learning [2] and distributed anomaly detection in sensor networks [3]. In such cases, analog OTA computation schemes can deliver sizable performance gains over classical separation-based approaches especially when the number of transmitters is large [2]-[7].

In some of the foreseen scenarios, such as e-health or industrial applications, security and privacy are expected to be major concerns in addition to efficient resource usage. Information theoretic secrecy can complement classic cryptography in addressing these issues. A natural way to enhance security in a setting such as in [4] is to add a jammer to the system that deteriorates the signal-to-noise ratio (SNR) of the eavesdropper and thereby prevents it from reconstructing a low-noise estimate of the objective function. In this case, it is necessary to place the jammer so that its signal is significantly stronger at the eavesdropper than it is at the legitimate receiver. Since in general, the exact position of an eavesdropper is not known, jammers typically have to be placed at multiple locations. In this work, we propose to turn this situation around and place the jammer so that its signal is stronger at the legitimate receiver than it is at the eavesdropper 1 . Such a setup is often easier to realize since the jammer can for instance be set up in proximity to the legitimate receiver

Part of this work was presented in [1] at the 2021 IEEE International Symposium on Information Theory, 12-20 July 2021, Melbourne, Victoria, Australia.

This work was supported by the German Research Foundation (DFG) within their priority program SPP 1798 "Compressed Sensing in Information Processing" and under grant STA 864/7-1. This work was also supported by the German Federal Ministry of Education and Research under grant 16KIS0605.

${ }^{1}$ For a formal statement of this condition, see the statement of Theorem 2 (or Theorem 1 for the special case of AWGN channels).

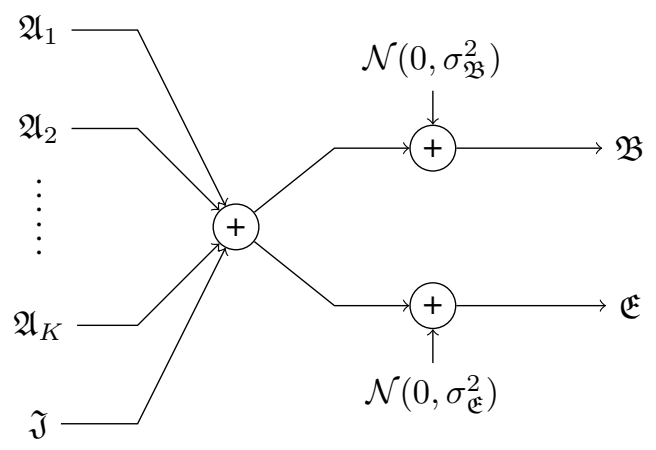

Fig. 1. AWGN channel model.

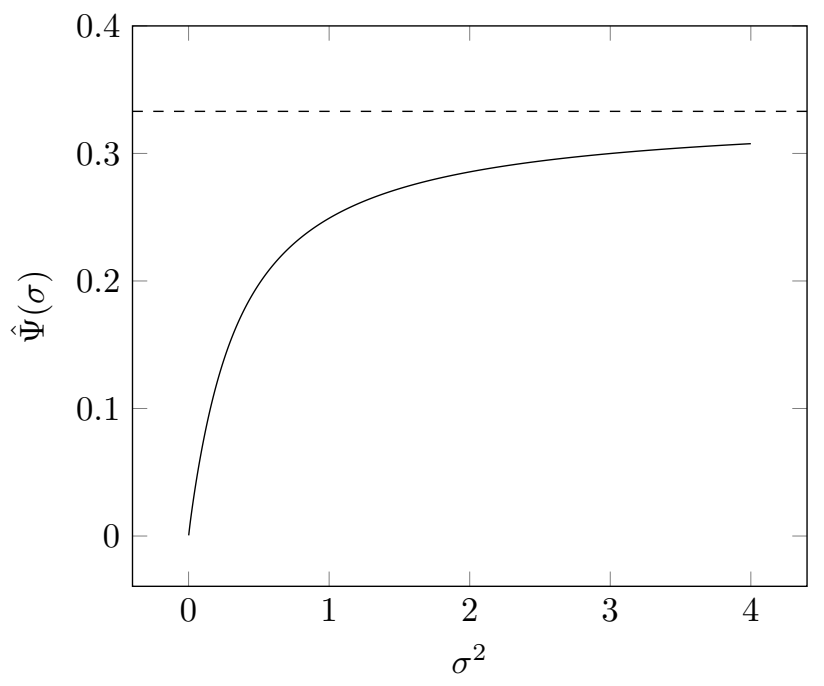

Fig. 2. Illustration of the MSE guarantees of Lemma 2 The dashed line is the MSE which an eavesdropper would have without any received signal (i.e., guessing the middle of the interval).

and in certain settings, such as factory buildings, it may be feasible to assume that the attacker is located, e.g., outside of the building while the legitimate receiver and the transmitters are inside. With this scenario in mind, our proposed scheme exploits the assumption that the jammer's signal is stronger at the legitimate receiver than it is at the eavesdropper. With that, it ensures that the eavesdropper observes a signal very similar to one jammed by white noise, while allowing the legitimate receiver to reconstruct the exact jamming sequence used and thus cancel it from its own received signal.

\section{A. Teaser: An AWGN Specialization of our Main Result}

Before proceeding to an overview of the main contributions of this work, we would like to give the reader an idea of 
the nature of our main result, since the formal statement in its full generality may not be immediately clear in terms of its applicability and will require the introduction of numerous preliminary definitions. In this subsection, therefore, we present an abridged and specialized form of our system model, problem statement and main result.

The system model consists of transmitters $\mathfrak{A}_{1}, \ldots, \mathfrak{A}_{K}$, a friendly jammer $\mathfrak{J}$, a legitimate receiver $\mathfrak{B}$, and an eavesdropper $\mathfrak{E}$.

Each $\mathfrak{A}_{k}$ holds a real number $s_{k}$, and the objective for $\mathfrak{A}_{1}, \ldots, \mathfrak{A}_{K}$ is to convey their numbers $s_{1}, \ldots, s_{K}$ to $\mathfrak{B}$ in such a way that $\mathfrak{B}$ cannot necessarily reconstruct all individual values, but rather a function $f\left(s_{1}, \ldots, s_{K}\right)$. While it is in principle possible to extend these methods to a larger function class (see [5]), in this paper we focus on the arithmetic mean function $f\left(s_{1}, \ldots, s_{K}\right):=\frac{1}{K} \sum_{k=1}^{K} s_{k}$ for values $s_{k} \in$ $[-1,1]$.

The transmitters, friendly jammer, and legitimate receiver are connected via a multiple-access wiretap channel with AWGN as depicted in Fig. 1, which also has a channel output for the eavesdropper $\mathfrak{E}$. The objective above has to be achieved while limiting the possibility of $\mathfrak{E}$ to learn too much about $f\left(s_{1}, \ldots, s_{K}\right)$.

Theorem 1. Consider a multiple-access wiretap channel as depicted in Fig. 1] and let each transmitter $\mathfrak{A}_{k}$ hold a value $s_{k} \in[-1,1]$. Assume further that $\sigma_{\mathfrak{E}}>\sigma_{\mathfrak{B}}$. Let $\mathfrak{P}_{\mathfrak{J}} \in(0, \infty)$. Then there are $\gamma_{1}, \gamma_{2} \in(0, \infty)$ such that we have for every $n \in \mathbb{N}$

- for each $k \in\{1, \ldots, K\}$, a pre-processor which takes $s_{k}$ as an input and outputs a random channel input sequence $T_{k}^{n}$ of average power at most 1 , and

- a random sequence $X^{n}$ of channel inputs for $\mathfrak{J}$ of average power at most $\mathfrak{P}_{\mathfrak{J}}$,

with the following properties:

- $\mathfrak{B}$ can reconstruct an estimate of $\frac{1}{K} \sum_{k=1}^{K} s_{k}$ with mean square error at most $\hat{\Psi}\left(\sigma_{\mathfrak{B}} / K\right)+\exp \left(-n \gamma_{1}\right)$, and

- any estimate of $\frac{1}{K} \sum_{k=1}^{K} s_{k}$ at $\mathfrak{E}$ has mean square error at least $\hat{\Psi}\left(\sqrt{\mathfrak{P}_{\mathfrak{J}}+\sigma_{\mathfrak{E}}^{2}} / K\right)-\exp \left(-n \gamma_{2}\right)$.

The function $\hat{\Psi}$ is strictly increasing and plotted in Fig. 2. It is defined as $\hat{\Psi}(\sigma):=\sigma^{2} \Psi(2 / \sigma)$ with $\Psi$ as given in (5).

Theorem 1 is a simplified and slightly specialized form of Corollary 2, which is stated and proven in Section V-B.

\section{B. Summary of the Main Contributions and Outline}

The main contributions of this paper are as follows:

1) We propose a novel framework and result for incorporating security considerations by including a friendly jammer in the system which deteriorates the eavesdropper's SNR while not impacting the legitimate receiver's ability to obtain an approximation of the function value which is to be OTA computed.

2) We show how this jamming strategy can be applied in the example case in which the channel is AWGN and the OTA computed function is an arithmetic average, and we prove that the security guarantee translates to a lower bound on the mean square error of the eavesdropper's function estimate.

3) We prove a theorem on compound channel coding for continuous alphabets. It is a generalization of the result of the part of [8] which considers finite Gaussian channels and we can consequently recover this result as a special case.

For 11 we use two information theoretic tools, namely coding for the compound channel and channel resolvability. The latter ensures that the jamming signal can be reconstructed fully by the legitimate receiver and thus be canceled from the received signal. Therefore, it has no impact on the quality of the objective function estimate. Channel resolvability guarantees that the jamming signal is virtually indistinguishable from white noise for the eavesdropper. This virtual indistinguishability is phrased in terms of variational distance between the actually observed distribution and a superposition of the transmitters' signal with white noise from the jammer. The coding result for the continuous compound channel which we derive may also be of interest as an independent result.

In Section I-C we survey the related literature. In Section we describe the system model considered in this work. We illustrate the idea with an application to the AWGN case in Section III before we give an overview of our main results in Section IV Section V details how our main result can be applied to classes of Gaussian channels, focusing in particular on the simple AWGN example from Section III In Section VI, we collect the detailed proofs of our results, and Section VII concludes the paper and states open questions for future research.

\section{Prior Work}

To the best of our knowledge, the OTA computation problem over a wiretap channel has not yet been considered in the literature. Therefore, in this subsection we briefly summarize the literature on the building blocks we use for the approach to the wiretap OTA computation channel that we propose in this work as well as for literature on concepts that are closely related to the ones presented in this paper.

a) OTA computation: The concept was originally introduced in [9]. In [3], [10], this idea was adapted to a setting where the domain and range of the function to be computed are continuous and the receiver can deal with a noisy estimate of the objective function value. In [4]-[6], we revisited this idea, adapted the existing scheme and provided an extension to a large class of functions and analysis of the estimation error for finite block length in a very general, fast-fading setting. There are many more prior works in this area. For details, we refer the reader to the literature section in [6].

b) Coding for compound channels: The compound channel problem was introduced independently in [11]-[13], while first independent results for the capacity expression can be found in [12], [13]. These works, however, explore mainly the case of finite input and output alphabets. The semi-continuous case in which only the input alphabet is assumed to be finite

${ }^{2}$ For a formal statement, see Definition 2 


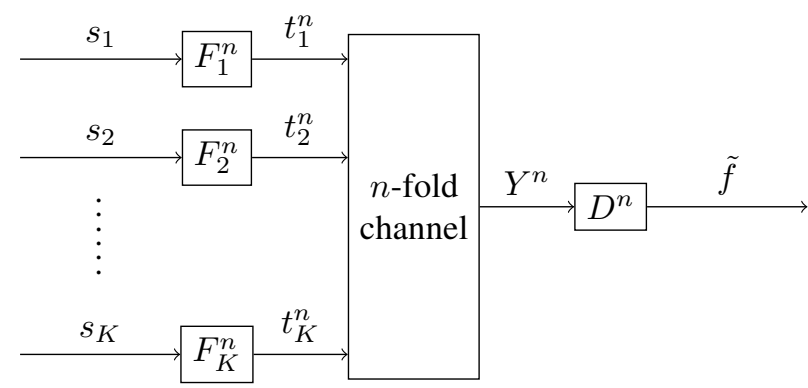

Fig. 3. System model for distributed function approximation described in Section II-A

is briefly touched upon in [13] and studied in more detail in [14] which provides an example showing that the capacity expression from the finite case does not carry over to the semicontinuous case in general. The semicontinuous case was further explored in [15], [16]. In many cases of practical interest, the capacity expression from the finite case can be generalized to the continuous case in which neither input nor output alphabets are assumed to be finite, as was found in [8] for a class of Gaussian compound channels.

c) Channel Resolvability and Semantic Security: The concept of channel resolvability was introduced in [17], [18]. Further results relevant in the context of this work appeared, e.g., in [19]-[22]. We use our generalization [23] for continuous channels as a basis for our proposed scheme. Although we cannot provide full semantic security guarantees in this work, we also heavily draw from the idea of obtaining semantic security by means of channel resolvability, which is layed out in [24]-[27].

d) Friendly Jamming: The idea of friendly jamming has been used in [28] to aid a transmitter-receiver pair in protecting a point-to-point transmission from a passive eavesdropper. Distributed and centralized beamforming techniques are used so that the jamming signal impacts the signal-to-noise ratio at the eavesdropper but not at the legitimate receiver. Several more recent works (cf., e.g., [29]-[31]) have expanded upon this idea and refined the friendly jamming techniques, but to the best of our knowledge, they have not yet been used to protect Over-the-Air computation against eavesdropping.

e) Physical Layer Security: The concept of information theoretic secrecy was introduced in [32] and the wiretap channel model together with a weaker, but more tractable notion of secrecy was introduced in [33]. Based on this, various stronger secrecy notions have been introduced and investigated (e.g., [26], [34], [35]). All of these existing works investigate how digitally coded transmissions can be protected against eavesdropping, while in the present work, we focus on uncoded analog transmissions over multiple-access channels.

\section{SYSTEM MODEL}

\section{A. Distributed Approximation of Functions}

In this subsection, we summarize the system model of the framework for analog OTA computation from [4]. This particular framework is called Distributed Function Approximation.
Let $\mathcal{S}_{1}, \ldots, \mathcal{S}_{K}$ be measurable spaces. The goal is to approximate functions $f: \mathcal{S}_{1} \times \ldots \times \mathcal{S}_{K} \rightarrow \mathbb{R}$ over a multiple-access channel $W$ with measurable input alphabets $\mathcal{X}_{1}, \ldots, \mathcal{X}_{K}$ and a measurable output alphabet $\mathcal{Y}$ in a distributed setting. An admissible distributed function approximation scheme for $f: \mathcal{S}_{1} \times \ldots \times \mathcal{S}_{K} \rightarrow \mathbb{R}$ for $n$ channel uses, depicted in Fig. 3, is a pair $\left(F^{n}, D^{n}\right)$, consisting of:

1) A pre-processing function $F^{n}=\left(F_{1}^{n}, \ldots, F_{K}^{n}\right)$ for the transmitters $\mathfrak{A}_{1}, \ldots, \mathfrak{A}_{K}$, where each $F_{k}^{n}$ is of the form

$$
F_{k}^{n}\left(s_{k}\right)=\left(t_{k, j}\left(s_{k}, U_{k}(j)\right)\right)_{j=1}^{n} \in \mathcal{X}_{k}^{n}
$$

with i.i.d. random variables $U_{k}(1), \ldots, U_{k}(n)$ and a measurable map

$$
\left(s_{k}, u_{1}, \ldots, u_{n}\right) \mapsto\left(t_{k, j}\left(s_{k}, u_{j}\right)\right)_{j=1}^{n} \in \mathcal{X}_{k}^{n} .
$$

2) A post-processing function $D^{n}$ for the receiver $\mathfrak{B}$ : The receiver is allowed to apply a measurable recovery function $D^{n}: \mathcal{Y}^{n} \rightarrow \mathbb{R}$ upon observing the output of the channel.

Note that this, contrary to the system model in [4], imposes the restriction that the pre-processing is i.i.d. across channel uses, which will be crucial for the security extension to the approximation scheme. Although this definition of admissible schemes is slightly less general, the scheme proposed in [4] still is an admissible scheme even in this stricter sense.

So in order to approximate $f$, the transmitters apply their pre-processing maps to

$$
\left(s_{1}, \ldots, s_{K}\right) \in \mathcal{S}_{1} \times \ldots \times \mathcal{S}_{K}
$$

resulting in $F_{1}^{n}\left(s_{1}\right), \ldots, F_{K}^{n}\left(s_{K}\right)$, which are sent over the channel. The receiver observes the output of the channel and applies the recovery map $D^{n}$. The whole process defines an estimate $\tilde{f}$ of $f$.

Depending on the application at hand, there are multiple ways in which the quality of the estimate $\tilde{f}$ can be quantified.

Definition 1. 1) Let $\delta, \varepsilon \in(0,1)$ and $f$ be given. We say that $f$ is $\delta$-approximated after $n$ channel uses with confidence level $\varepsilon$ if there is an approximation scheme $\left(F^{n}, D^{n}\right)$ such that the resulting estimate $\tilde{f}$ of $f$ satisfies

$$
\mathbb{P}\left(\left|\tilde{f}-f\left(s_{1}, \ldots, s_{K}\right)\right| \geq \delta\right) \leq \varepsilon
$$

for all $s^{K}:=\left(s_{1}, \ldots, s_{K}\right) \in \mathcal{S}_{1} \times \ldots \times \mathcal{S}_{K}$.

2) We say that $f$ is V-MSE-approximated if, under a uniform distribution of $f\left(s_{1}, \ldots, s_{K}\right)$, we have

$$
\mathbb{E}\left(\left(\tilde{f}-f\left(s_{1}, \ldots, s_{K}\right)\right)^{2}\right) \leq V
$$

where the expectation is over the joint distribution of $s_{1}, \ldots, s_{K}$ and $\tilde{f}$ which is induced by the distributed function approximation scheme and the channel.

Distributed Function Approximation schemes have to be tailored to the structure of $W$. It is worth noting, however, that for large classes of wireless channel models and objective functions, such schemes exist. In [4], we have proposed a Distributed Function Approximation scheme for fast-fading wireless channels with sub-Gaussian fading and noise, where 


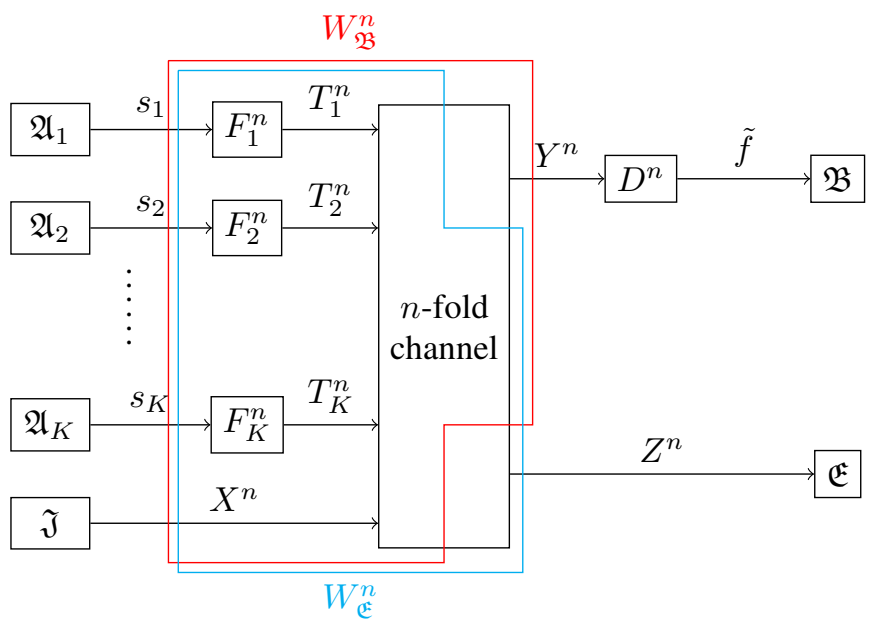

Fig. 4. System model for distributed function approximation with jamming described in Section II-B

the quality of approximation is defined as in item 1 of Definition [1 In [5], [6], we have extended the scheme to cases of correlated fading and noise. This means that the proposed scheme is applicable to fading wireless channels even in the presence of non-Gaussian and bursty interference and noise, and for various levels of mobility and richness of the scattering environment. However, a major limitation of these works is that the distributed data has to be transmitted without the possibility to encrypt or otherwise protect it against eavesdropping.

\section{B. Secrecy Extension to Distributed Approximation of Func-} tions

In order to incorporate security aspects into the framework, we consider the extended system model depicted in Fig. 4.

The first addition to the model is an attacker $\mathfrak{E}$ which attempts to eavesdrop on the transmission and wants to gain knowledge about $s_{1}, \ldots, s_{K}$. At each channel use, $\mathfrak{E}$ observes an output $Z$ ranging over the eavesdropper's alphabet $\mathcal{Z}$. As a counter-measure, we add a friendly jammer $\mathfrak{J}$ which transmits some jamming sequence $X^{n}$ with the objective to prevent $\mathfrak{E}$ from obtaining information while still allowing $\mathfrak{B}$ to obtain a good estimate of $f\left(s_{1}, \ldots, s_{K}\right)$.

Definition 2. A distributed function approximation scheme with jamming consists of:

- A distributed function approximation scheme as described in Section II-A. i.e., pre- and post-processing schemes

- A jamming strategy given by a probability distribution on $\mathcal{X}^{n}$.

We say that a distributed function approximation scheme with jamming allows reconstruction of the jamming signal with probability $\epsilon$ if there is a decoding function $\vartheta: \mathcal{Y}^{n} \rightarrow \mathcal{X}^{n}$ such that

$$
\sup _{s_{1} \in \mathcal{S}_{1}, \ldots, s_{K} \in \mathcal{S}_{K}} \mathbb{P}_{s_{1}, \ldots, s_{K}}\left(\vartheta\left(Y^{n}\right) \neq X^{n}\right) \leq \epsilon
$$

and $\epsilon$ is the smallest number with this property.
The objective is to find admissible pre- and post-processing strategies as well as a jamming strategy such that $\mathfrak{B}$ can obtain a good approximation $\tilde{f}$ of $f\left(s_{1}, \ldots, s_{K}\right)$ while bounding the usefulness of any information that $\mathfrak{E}$ can obtain about $s_{1}, \ldots, s_{K}$.

Together with the channel, a distributed function approximation scheme with jamming induces a probability distribution $\hat{R}_{s_{1}, \ldots, s_{K}}$ on $\mathcal{Z}^{n}$ for each $\left(s_{1}, \ldots, s_{K}\right) \in \mathcal{S}$. How secure the scheme is depends on how strongly $\hat{R}_{s_{1}, \ldots, s_{K}}$ depends on $s_{1}, \ldots, s_{K}$. In the following, we formalize this notion.

Any measurable function $g: \mathcal{S} \rightarrow \mathcal{T}$, where $\mathcal{T}$ is a measurable space, is called an eavesdropper's objective.

Definition 3. 1) Given a real number $\delta \geq 0$, we say that a distributed function approximation scheme with jamming is $\delta$-semantically secure if there is a probability measure $\mu$ on $\mathcal{Z}^{n}$ such that for all $\left(s_{1}, \ldots, s_{K}\right) \in \mathcal{S}$,

$$
\left\|\hat{R}_{s_{1}, \ldots, s_{K}}-\mu\right\|_{\mathrm{TV}} \leq \delta
$$

where $\|\cdot\|_{\mathrm{TV}}$ denotes the total variation norm on signed measures.

2) Let $g: \mathcal{S} \rightarrow \mathcal{T}$, where $\mathcal{T} \subseteq \mathbb{R}$ is measurable and bounded, be an eavesdropper's objective. Let $V \geq 0$ be a real number. We say that a distributed function approximation scheme with jamming is $(g, V)$-MSEsecure if under a uniform distribution of $g\left(s_{1}, \ldots, s_{K}\right)$, for every estimator $d: \mathcal{Z}^{n} \rightarrow \mathcal{T}$, we have

$$
\mathbb{E}\left(\left(d\left(Z^{n}\right)-g\left(s_{1}, \ldots, s_{K}\right)\right)^{2}\right) \geq V,
$$

where the expectation is over the joint distribution of $s_{1}, \ldots, s_{K}$ and $Y^{n}$ which results from the application of the distributed function approximation scheme with jamming and the channel.

In statistical terms, that a scheme is $(g, V)$-MSE-secure means that all estimators the eavesdropper can apply have mean square error (MSE) at least $V$ under a uniformly distributed objective. A uniform distribution of the objective means that $s_{1}, \ldots, s_{K}$ are randomly distributed in such a way that $g\left(s_{1}, \ldots, s_{K}\right)$ follows a uniform distribution.

In a sense made explicit by the following lemma, semantic security is the stronger of the two security notions from Definition 3

Lemma 1. Let $\mathcal{T}:=[a, b]$, let $g: \mathcal{S} \rightarrow \mathcal{T}$ be an eavesdropper's objective and $\delta \geq 0$ a real number.

Then, any distributed function approximation scheme with jamming that is $\delta$-semantically secure is also $(g,(1 / 12-\delta)(b-$ $\left.a)^{2}\right)$-MSE-secure.

Proof. Let $d: \mathcal{Z}^{n} \rightarrow \mathcal{T}$. Then, assuming the distribution of $s_{1}, \ldots, s_{K}$ corresponds to a uniform distribution on $[a, b]$ of 


$$
\begin{aligned}
& g\left(s_{1}, \ldots, s_{K}\right), \text { we have } \\
& \quad \mathbb{E}_{s_{1}, \ldots, s_{K}} \mathbb{E}_{\hat{R}_{s_{1}, \ldots, s_{K}}}\left(\left(d\left(Z^{n}\right)-g\left(s_{1}, \ldots, s_{K}\right)\right)^{2}\right) \\
& =\mathbb{E}_{s_{1}, \ldots, s_{K}} \int_{0}^{(b-a)^{2}} \hat{R}_{s_{1}, \ldots, s_{K}}\left(\left(d\left(Z^{n}\right)\right.\right. \\
& \left.\left.\quad-g\left(s_{1}, \ldots, s_{K}\right)\right)^{2}>t\right) d t \\
& \quad 11 \mathbb{E}_{s_{1}, \ldots, s_{K}} \int_{0}^{(b-a)^{2}}\left(\mu \left(\left(d\left(Z^{n}\right)-g\left(s_{1}, \ldots, s_{K}\right)\right)^{2}\right.\right. \\
& =\mathbb{E}_{s_{1}, \ldots, s_{K}} \mathbb{E}_{\mu}\left(\left(d\left(Z^{n}\right)-g\left(s_{1}, \ldots, s_{K}\right)\right)^{2}\right)-\delta(b-a)^{2} \\
& \geq\left(\frac{1}{12}-\delta\right)(b-a)^{2},
\end{aligned}
$$

where the last step is because under $\mu, Z^{n}$ is independent of $s_{1}, \ldots, s_{K}$. Therefore, the posterior distribution of $g\left(s_{1}, \ldots, s_{K}\right)$ given $Z^{n}$ is uniform on $[a, b]$, which implies that the minimum MSE is the variance of the uniform distribution. Denoting with $U$ a random variable distributed uniformly in $[a, b]$, we can calculate its variance as

$$
\begin{aligned}
\mathbb{E}\left(U^{2}\right)-(\mathbb{E} U)^{2} & =\int_{a}^{b} \frac{u^{2}}{b-a} d u-\frac{(a+b)^{2}}{4} \\
& =\frac{b^{3}-a^{3}}{3(b-a)}-\frac{(a+b)^{2}}{4} \\
& =\frac{b^{2}+a^{2}+a b}{3}-\frac{a^{2}+2 a b+b^{2}}{4} \\
& =\frac{4 b^{2}+4 a^{2}+4 a b-3 a^{2}-6 a b-3 b^{2}}{12} \\
& =\frac{1}{12}(b-a)^{2}
\end{aligned}
$$

\section{Special case $K=1$}

We conclude this section with a brief discussion of the important special case $K=1$. While one of the main motivations of the methods developed in this paper is their scalability to large values of $K$, the case of low values of $K$ can also be interesting in many practical applications and be instructive to understand the nature of our results better.

For the special case of only a single transmitter $(K=1)$, the problem reduces to a point-to-point transmission of the real number $f\left(s_{1}\right)$ in the presence of an eavesdropper and a friendly jammer. In our results in this paper, there is no assumption that $K$ has to be large; in particular, they remain applicable also when $K=1$. However, since in this case no function of distributed values has to be computed over the channel, it is possible to separately source and channel encode $f\left(s_{1}\right)$. After the source coding step has been performed, the remaining problem is very similar to jammer-aided secret communication as treated for instance in [28]-[30].

But although this approach is applicable to the same communication task, it is important to note that the way in which the friendly jammer has to be placed differs significantly. In the approach of this paper, the jamming signal has to be stronger at the eavesdropper than it is at the legitimate receiver. As long as this condition is satisfied, the legitimate receiver has the ability to almost completely cancel the jamming signal. This means that our method remains applicable even if the gap in terms of jammer signal strength between the legitimate receiver and the eavesdropper is relatively small. In [28]-[30], on the other hand, it is necessary that the jamming signal is stronger at the eavesdropper than it is at the legitimate receiver. Moreover, this gap between signal strengths has to be as large as possible since the jammer's signal strength at the legitimate receiver diminishes the capacity of the main channel. Therefore, our results in this case are more suitable for scenarios where is is possible to assure a high jamming signal strength at the legitimate receiver while results from [28]-[30] are more suitable in cases where all possible eavesdropper locations can be covered with strong jamming signals that have very low strength at the location of the legitimate receiver.

With respect to the open research questions given in Section VII, we remark that methods from the literature can be used to achieve semantic security with slight adaptations; such a construction is for instance sketched in [36]. We are not aware of practically feasible schemes that achieve semantic security, but we expect that weaker guarantees such as MSE security could be derived, e.g., for the approach given in [37]. In order to accommodate a friendly jammer in the system model, all of these approaches would need to be combined with the works on friendly jamming discussed above. Therefore, it would remain necessary to also have the assumption that the jamming signal is significantly stronger at the eavesdropper than it is at the legitimate receiver. For the case in which this assumption is reversed as in the present work, to the best of our knowledge these questions remain open even for $K=1$.

\section{Specialization to the AWGN Channel}

In general, the approximation scheme even without an eavesdropper or jammer highly depends on the particular structure of the channel and $f$. It is therefore instructive to consider a specialization of the Distributed Function Approximation framework with jamming to the computation of arithmetic means over AWGN channels. Specifically, the objective function is given as

$$
f:\left(s_{1}, \ldots, s_{K}\right) \mapsto \frac{1}{K} \sum_{k=1}^{K} s_{k},
$$

where for all $k, \mathcal{S}_{k}=[-1,1]$. The channel is given by

$$
\begin{aligned}
Y & =h_{\mathfrak{A} \mathfrak{B}} \sum_{k=1}^{K} T_{k}+h_{\mathfrak{J} \mathfrak{B}} X+N_{\mathfrak{B}} \\
Z & =h_{\mathfrak{A E}} \sum_{k=1}^{K} T_{k}+h_{\mathfrak{J} \mathfrak{E}} X+N_{\mathfrak{E}} .
\end{aligned}
$$

$N_{\mathfrak{B}}$ is centered normal with variance $\sigma_{\mathfrak{B}}^{2}$ and $N_{\mathfrak{E}}$ is centered normal with variance $\sigma_{\mathfrak{E}}^{2}$. The real channel coefficients $h_{\mathfrak{A} \mathfrak{B}}, h_{\mathfrak{J} \mathfrak{B}}, h_{\mathfrak{A E}}, h_{\mathfrak{J E}}$ are assumed deterministic and known everywhere. The channel is used $n$ times with transmitter 
input sequences $T_{k}^{n}$ for each $k \in\{1, \ldots, K\}$ and $X^{n}$ for the jammer. The input sequences are subject to the average power constraints

$$
\frac{1}{n} \sum_{j=1}^{n}\left(T_{k, j}\right)^{2} \leq \mathfrak{P}_{\mathfrak{A}}, \quad \frac{1}{n} \sum_{j=1}^{n}\left(X_{i}\right)^{2} \leq \mathfrak{P}_{\mathfrak{J}}
$$

The problem is easily approached if we can assume that $\mathfrak{B}$ has full knowledge of $X^{n}$, while $\mathfrak{E}$ knows only how $X^{n}$ is distributed.

In this case, we have the following result.

Lemma 2. Consider the wiretap channel given by (3) and (4) and the objective function $f$ defined in (2). Define

$$
\sigma_{\text {eff, } \mathfrak{B}}^{2}:=\frac{\sigma_{\mathfrak{B}}^{2}}{h_{\mathfrak{A} \mathfrak{B}}^{2} K^{2} \mathfrak{P}_{\mathfrak{A}}}, \quad \sigma_{\text {eff, }}^{2}:=\frac{\sigma_{\mathfrak{E}}^{2}+h_{\mathfrak{J} \mathfrak{E}}^{2} \mathfrak{P}_{\mathfrak{J}}}{h_{\mathfrak{A} \mathfrak{E}}^{2} K^{2} \mathfrak{P}_{\mathfrak{A}}}
$$

Assume that the jamming sequence $X^{n}$ is perfectly known at the legitimate receiver while the eavesdropper has only statistical information. Define

$$
\begin{array}{r}
\Psi(t):=\int_{0}^{t} \int_{-\infty}^{\infty}\left(v+\frac{\varphi_{N}(-v)-\varphi_{N}(t-v)}{\Phi_{N}(t-v)-\Phi_{N}(-v)}-u\right)^{2} \\
\cdot \frac{1}{t} \varphi_{N}(u-v) d v d u
\end{array}
$$

where $\varphi_{N}$ denotes the probability density function and $\Phi_{N}$ the cumulative distribution function of the standard normal distribution, respectively. Then there is a distributed function approximation scheme with jamming which is $\left(f, \sigma_{\text {eff,EE }}^{2} \Psi\left(2 / \sigma_{\text {eff, }}\right)\right)$-MSE-secure and $\left(\sigma_{\text {eff, } \mathfrak{B}}^{2} \Psi\left(2 / \sigma_{\text {eff, } \mathfrak{B}}\right)\right)$ $M S E$-approximates $f$ at the receiver.

In order to understand the impact of the function $\Psi$ that appears in the security and approximation guarantees, we refer the reader to the plot of the function $\hat{\Psi}: \sigma^{2} \mapsto \sigma^{2} \Psi(2 / \sigma)$ in Fig. 2

The proof of Lemma 2 is based on a few facts from statistics. We only state the relevant lemmas here. For the sake of completeness, we include the proofs of the following two lemmas in Section VI-A

Lemma 3. If $U$ is distributed uniformly in $[a, b]$ and, conditioned on $U, V_{1}, \ldots, V_{n}$ are i.i.d. normally distributed with mean $U$ and variance $\sigma^{2}$, then the minimum MSE estimator for estimating $U$ from the observations $V_{1}, \ldots, V_{n}$ is

$$
\hat{U}:=\bar{V}+\frac{\sigma}{\sqrt{n}} \cdot \frac{\varphi_{N}\left(\frac{a-\bar{V}}{\sigma / \sqrt{n}}\right)-\varphi_{N}\left(\frac{b-\bar{V}}{\sigma / \sqrt{n}}\right)}{\Phi_{N}\left(\frac{b-\bar{V}}{\sigma / \sqrt{n}}\right)-\Phi_{N}\left(\frac{a-\bar{V}}{\sigma / \sqrt{n}}\right)},
$$

where $\bar{V}:=\frac{1}{n} \sum_{j=1}^{n} V_{j}$.

Lemma 4. Under the assumptions of Lemma 3 the estimator $\hat{U}$ satisfies

$$
\mathbb{E}\left((U-\hat{U})^{2}\right)=\frac{\sigma^{2}}{n} \Psi\left(\frac{b-a}{\sigma / \sqrt{n}}\right),
$$

with $\Psi$ as defined in (5).
Proof of Lemma 2. We use the following transmission strategy:

$$
\begin{aligned}
& X_{j}: \text { Gaussian with mean } 0 \text { and variance } \mathfrak{P}_{\mathfrak{J}}, \\
& F_{k}^{n}: s_{k} \mapsto(1, \ldots, 1) \cdot s_{k} \sqrt{\frac{\mathfrak{P}_{\mathfrak{A}}}{n}}
\end{aligned}
$$

The receiver can obtain

$$
\begin{aligned}
Y_{j}^{\prime} & :=\frac{Y_{j}-h_{\mathfrak{J} \mathfrak{B}} X_{j}}{h_{\mathfrak{A} \mathfrak{B}} K \sqrt{\mathfrak{P}_{\mathfrak{A}} / n}}=\frac{h_{\mathfrak{A} \mathfrak{B}} \sum_{k=1}^{K} T_{k}+N_{\mathfrak{B}, j}}{h_{\mathfrak{A} \mathfrak{B}} K \sqrt{\mathfrak{P}_{\mathfrak{A}} / n}} \\
& =\frac{h_{\mathfrak{A} \mathfrak{B}} \sum_{k=1}^{K} s_{k} \sqrt{\mathfrak{P}_{\mathfrak{A}} / n}+N_{\mathfrak{B}, j}}{h_{\mathfrak{A} \mathfrak{B}} K \sqrt{\mathfrak{P}_{\mathfrak{A}} / n}} \\
& =f\left(s_{1}, \ldots, s_{K}\right)+\frac{N_{\mathfrak{B}, j}}{h_{\mathfrak{A} \mathfrak{B}} K \sqrt{\mathfrak{P}_{\mathfrak{A}} / n}} .
\end{aligned}
$$

We define the post-processing operation $D^{n}$ at the receiver as first obtaining $Y_{1}^{\prime}, \ldots, Y_{n}^{\prime}$ and then computing the minimum MSE estimator from Lemma 3. With this choice, Lemma 4 yields the claimed reconstruction error guarantee.

On the other hand, the output at $\mathfrak{E}$ is given by

$$
\begin{aligned}
Z_{j} & \stackrel{\text { 年 }}{=} h_{\mathfrak{A E}} \sum_{k=1}^{K} T_{k}+h_{\mathfrak{J E}} X_{j}+N_{\mathfrak{E}, j} \\
& \stackrel{\text { 8 }}{=} h_{\mathfrak{A E}} \sum_{k=1}^{K} s_{k} \sqrt{\mathfrak{P}_{\mathfrak{A}} / n}+h_{\mathfrak{J E}} X_{j}+N_{\mathfrak{E}, j} \\
& \stackrel{\text { 2 }}{=} f\left(s_{1}, \ldots, s_{K}\right) \cdot K \sqrt{\mathfrak{P}_{\mathfrak{A}} / n} h_{\mathfrak{A E}}+h_{\mathfrak{J} \mathfrak{E}} X_{j}+N_{\mathfrak{E}, j} .
\end{aligned}
$$

From this, Lemmas 3 and 4 yield the claimed MSE-security of the scheme.

The assumption that the legitimate receiver has full knowledge of the jamming signal seems quite strong. So the main part of this paper is devoted to setting out and analyzing a jamming strategy in Theorem 2 which does not need any form of shared randomness or additional communication between the friendly jammer and the legitimate receiver. This jamming strategy has "almost" the same implications on the overall system performance as the assumption that the legitimate receiver has full knowledge of the jamming signal, while the eavesdropper only has knowledge about its distribution. In Corollary 2, we formalize this notion for the AWGN case. In principle, however, this jamming strategy is not restricted to the AWGN scenario; but in fact, it can be combined with any class of channel models in which it is possible to cancel or at least mitigate the effect of the jamming signal if exact knowledge of it is available.

One example where not a full cancellation but at least a good mitigation is possible is the fast-fading scenario introduced in [4].

\section{Main Results}

In this section, we formally state the main results of this work.

A function approximation scheme is specific to a particular channel model, which among other things influences how the pre- and post-processing operations have to be designed as well as which class of functions can be approximated. In 
Section [II], we have described such a scheme for the AWGN channel and only a singleton class of functions, namely for the arithmetic average, which is a particularly simple case. The strategy for the legitimate receiver to counter the signal of the friendly jammer given that it is known is also particularly simple in the AWGN case and can be done perfectly, as we have seen. The missing part of the secrecy extension, which is the method for the legitimate receiver to obtain the necessary knowledge of the jamming signal while the eavesdropper cannot, on the other hand, can be phrased and proven to work in somewhat greater generality. In order to formally state our main results, therefore, we have to introduce a few technical concepts first.

For any channel $W$, we denote the joint input-output distribution under $P$ and $W$ by $Q_{P, W}$ and the marginal for $\mathcal{Y}$ by $R_{P, W}$. With these conventions, we define the information density of tuples of elements of the input and output alphabets under the channel $W$ and an input distribution $P$ as

$$
\mathbf{i}_{P, W}\left(x^{n} ; y^{n}\right):=\log \frac{d W^{n}\left(x^{n}, \cdot\right)}{d R_{P, W}^{n}}\left(y^{n}\right) .
$$

Here and for the remainder of the paper, the functions exp and log both use Euler's number as their basis, and all information quantities are consequently given in nats.

Correspondingly, the mutual information is defined as

$$
\mathbf{I}_{P, W}:=\mathbb{E}_{Q_{P, W}} \mathbf{i}_{P, W}(X ; Y) .
$$

Moreover, given two probability measures $\mu$ and $\nu$, we define the Rényi divergence of order $\alpha \in(0,1) \cup(1, \infty)$ between them as

$$
\mathbf{D}_{\alpha}(\mu \| \nu):=\frac{1}{\alpha-1} \log \mathbb{E}_{\mu}\left(\left(\frac{d \mu}{d \nu}\right)^{\alpha-1}\right) .
$$

$\mathbf{D}_{1}(\mu \| \nu):=\lim _{\alpha \succ 1} \mathbf{D}_{\alpha}(\mu \| \nu)$ is the Kullback-Leibler divergence.

A compound channel is a family $\left(W_{s}\right)_{s \in \mathcal{S}}$ of memoryless time-discrete point-to-point channels with common input alphabet $\mathcal{X}$ and output alphabet $\mathcal{Y}$. The transmitter's channel input is passed through a fixed $W_{s}$ for the entire block length, but the transmitter does not control the choice of $s$, nor is it governed by a probability distribution. In this work, we assume neither the transmitter nor the receiver knows $s$. A compound channel code with block length $n$ and rate $\mathcal{R}$ consists of an encoder $e:\{1, \ldots, \exp (n \mathcal{R})\} \rightarrow \mathcal{X}^{n}$ and a decoder $d: \mathcal{Y}^{n} \rightarrow\{1, \ldots, \exp (n \mathcal{R})\}$. We say that it has error probability $\epsilon$ if under a uniform distribution of $\mathcal{M} \in\{1, \ldots, \exp (n \mathcal{R})\}$, the following is true: Let $Y^{n}$ be constructed by passing the components of $X^{n}:=e(\mathcal{M})$ independently through $W_{s}$. Then, we have

$$
\sup _{s \in \mathcal{S}} \mathbb{E}_{\mathcal{M}} \mathbb{P}_{s}\left(m \neq d\left(Y^{n}\right)\right) \leq \epsilon,
$$

where $\epsilon$ is the smallest number with this property.

Our secrecy scheme will hinge on the capacity of compound channels with possibly continuous alphabets (such as Gaussian compound channels). As mentioned in Section $[$ it is shown in [14] that even in the case that only the output alphabet is countably infinite, the capacity expressions from the finite case [12], [13] do not carry over. It is therefore clear that an additional assumption on the compound channel is needed. In existing literature (e.g., [8], [12]), the problem is often approached by proving that the compound channel can be approximated by a finite class of channels in which case classical channel coding techniques such as joint typicality decoding can be adapted in a straightforward manner. In this work, we choose to directly pose the approximability of the compound channel by a finite class of channels as an assumption of our coding theorem. In Section $\mathrm{V}-\mathrm{A}$, we justify the usefulness of results involving this assumption by proving that a large class of practically relevant channels can indeed be approximated in the sense of the following definition.

Given measures $\mu$ and $\nu$, we say that $\mu$ is absolutely continuous with respect to $\nu$, or $\mu \ll \nu$, if all $\nu$-null sets are also $\mu$-null sets.

Definition 4. Given a compound channel $\left(W_{s}\right)_{s \in \mathcal{S}}$ with input alphabet $\mathcal{X}$ and output alphabet $\mathcal{Y}$, we say that it can be $(\delta, J)$-approximated under a probability distribution $P$ on $\mathcal{X}$ if there is a sequence $\left(\hat{W}_{\delta, j}\right)_{j=1}^{J}$ of channels from $\mathcal{X}$ to $\mathcal{Y}$ such that for every $s \in \mathcal{S}$, there is $j \in\{1, \ldots, J\}$ such that

$$
\begin{aligned}
& \mathbb{E}_{P} \mathbf{D}_{1}\left(W_{s}(X, \cdot) \| \hat{W}_{\delta, j}(X, \cdot)\right) \leq \delta \\
\exists \alpha>1 \forall x \in \mathcal{X}: & \mathbf{D}_{\alpha}\left(W_{s}(x, \cdot) \| \hat{W}_{\delta, j}(x, \cdot)\right)<\infty \\
\forall x \in \mathcal{X}: \quad & \hat{W}_{\delta, j}(x, \cdot) \ll W_{s}(x, \cdot) \\
& \mathbf{I}_{P, \hat{W}_{\delta, j}}-\mathbf{I}_{P, W_{s}} \leq \delta,
\end{aligned}
$$

and for every $j \in\{1, \ldots, J\}$ there is $s \in \mathcal{S}$ such that

$$
\mathbf{I}_{P, W_{s}}-\mathbf{I}_{P, \hat{W}_{\delta, j}} \leq \delta .
$$

The discussion in Section $\nabla-\mathrm{A}$ provides sufficient topological conditions for $(\delta, J)$-approximability and show that an example of channels with this property are (possibly fading) Gaussian channels.

We use a standard random codebook construction: Given a channel input alphabet $\mathcal{X}$, a distribution $P$ on $\mathcal{X}$, a block length $n$ and a rate $\mathcal{R}$, we define the $(P, n, \mathcal{R})$-ensemble of codebooks as a random experiment in which $\exp (n \mathcal{R})$ codewords of length $n$ are drawn randomly and independently according to $P$ for each component of each codeword.

A codebook $\mathcal{C}$ induces a jamming strategy in the following way: The jammer draws a codeword index $\mathcal{M}$ uniformly at random and transmits $\mathcal{C}(\mathcal{M})$, the codeword in $\mathcal{C}$ indexed by $\mathcal{M}$. Therefore, the number of codewords in the codebook controls the amount of randomness contained in the jamming signal.

In order to be able to impose an average power constraint on the jammer, we define an additive cost constraint $(c, C)$ for an input alphabet $\mathcal{X}$ consisting of a function $c: \mathcal{X} \rightarrow \mathbb{R}_{0}^{+}$ and a number $C \in \mathbb{R}_{0}^{+}$. Given any $n$, we say that $x^{n} \in \mathcal{X}^{n}$ satisfies the cost constraint if $\sum_{j=1}^{n} c\left(x_{j}\right) \leq n C$.

The specialization of this definition to a usual average power constraint would be to pick the square function as $c$ and the maximum admissible average power as $C$.

As long as there is at least one $x^{n} \in \mathcal{X}^{n}$ which satisfies the cost constraint $(c, C)$, given any codebook $\mathcal{C}$ of block length 
$n$, we can define an associated cost-constrained codebook $\mathcal{C}_{c, C}$ which is generated from $\mathcal{C}$ by replacing all codewords that do not satisfy the cost constraint with $x^{n}$. Obviously, all codewords in a cost-constrained codebook satisfy the cost constraint. We say that a cost constraint $(c, C)$ is compatible with an input distribution $P$ if for a random variable $X$ distributed according to $P, c(X)$ has a finite moment generating function in an interval containing 0 in its interior and $C>\mathbb{E}_{P} c(X)$.

We assume a given pre-processing scheme which is admissible as defined in Section II-A and consider effective channels incorporating both the pre-processing at the transmitters and the physical channel. We denote the legitimate user's effective channel, which is a stochastic kernel mapping from $\mathcal{S}_{1} \times \cdots \times \mathcal{S}_{K} \times \mathcal{X}$ to $\mathcal{Y}$, by $W_{\mathfrak{B}}$ and the eavesdropper's effective channel, which is a stochastic kernel mapping from $\mathcal{S}_{1} \times \cdots \times \mathcal{S}_{K} \times \mathcal{X}$ to $\mathcal{Z}$, by $W_{\mathfrak{E}}$. The $n$-fold products of these effective channels are outlined in the system model in Fig. 4 With these concepts and notations defined, we are ready to state the main result of this work, which gives sufficient conditions for the existence of a jamming scheme that can simultaneously ensure that the legitimate receiver is able to reconstruct the full jamming signal and limit the usefulness of the eavesdropper's received signal.

Theorem 2. Let $P$ be a jammer input distribution. Suppose that for every $\delta>0$, there is some $J(\delta)$ such that the compound channel $\left(W_{s}\right)_{s \in \mathcal{S}}$ defined by $W_{\left(s_{1}, \ldots, s_{K}\right)}:=$ $W_{\mathfrak{B}}\left(s_{1}, \ldots, s_{K}, \cdot, \cdot\right)$ can be $(\delta, J(\delta))$-approximated under $P$. Suppose further that for all $s_{1} \in \mathcal{S}_{1}, \ldots, s_{K} \in \mathcal{S}_{K}$, the moment-generating function

$$
\mathbb{E} \exp \left(t \cdot \mathbf{i}_{P, W_{\mathfrak{E}}\left(s_{1}, \ldots, s_{K}, \cdot, \cdot\right)}(X ; Z)\right)
$$

of the information density exists and is finite at some point $t>0$. Let $(c, C)$ be an additive cost constraint compatible with $P$, and let $\mathcal{C}$ be a random codebook from the $(P, n, \mathcal{R})$ ensemble. Let $\mathcal{R} \in(0, \infty)$ such that

$$
\begin{aligned}
\sup _{s_{1} \in \mathcal{S}_{1}, \ldots, s_{K} \in \mathcal{S}_{K}} & \mathbf{I}_{P, W_{\mathfrak{E}}\left(s_{1}, \ldots, s_{K}, \cdot, \cdot\right)}<\mathcal{R} \\
& <\inf _{s_{1} \in \mathcal{S}_{1}, \ldots, s_{K} \in \mathcal{S}_{K}} \mathbf{I}_{P, W_{\mathfrak{B}}\left(s_{1}, \ldots, s_{K}, \cdot, \cdot\right)} .
\end{aligned}
$$

Then there are numbers $\gamma_{1}, \gamma_{2}, \gamma_{3}, \gamma_{4}>0$ such that for sufficiently large $n$,

$$
\begin{array}{r}
\mathbb{P}_{\mathcal{C}}\left(\left\|\hat{R}_{W_{\mathfrak{E}}\left(s_{1}, \ldots, s_{K}, \cdot, \cdot\right)^{n}, \mathcal{C}_{c, C}}-R_{P, W_{\mathfrak{E}}\left(s_{1}, \ldots, s_{K}, \cdot, \cdot\right)}^{n}\right\|_{\mathrm{TV}}\right. \\
\left.\geq \exp \left(-n \gamma_{1}\right)\right)<\exp \left(-\exp \left(n \gamma_{2}\right)\right)
\end{array}
$$

and

$$
\mathbb{P}_{\mathcal{C}}(\mathcal{E})<\exp \left(-n \gamma_{4}\right),
$$

where $\mathcal{E}$ is the event that the jamming strategy induced by $\mathcal{C}_{c, C}$ does not allow reconstruction of the jamming signal with error at most $\exp \left(-n \gamma_{3}\right)$.

Obviously, the theorem is only useful if there exists some $\mathcal{R}$ satisfying (14). The condition that such an $\mathcal{R}$ exists is the formalization of the notion that the jamming signal has to be stronger at the legitimate receiver than it is at the eavesdropper.
In Section $\mathrm{V}$-B we discuss in more detail how the guarantee in (15) can be used to arrive at a MSE security guarantee for the scheme.

Theorem 2 needs a compound channel coding result as an ingredient for its proof, and since this result is slightly more general than results available in the literature, it may be of independent interest. Therefore, we also state it in this section.

Theorem 3. Let $\left(W_{s}\right)_{s \in \mathcal{S}}$ be a compound channel with input alphabet $\mathcal{X}$ and output alphabet $\mathcal{Y}$, and let $P$ be a probability distribution on $\mathcal{X}$ such that for every $\delta>0$, there is a $J(\delta)$ such that $\left(W_{s}\right)_{s \in \mathcal{S}}$ can be $(\delta, J(\delta))$-approximated under $P$. Let

$$
0<\mathcal{R}<\inf _{s \in \mathcal{S}} \mathbf{I}_{P, W_{s}}
$$

and let $\mathcal{C}$ be a random codebook from the $(P, n, \mathcal{R})$-ensemble. Define an encoder $m \mapsto \mathcal{C}(m)$. Then there is a decoder such that the error probability $\epsilon$ of the resulting compound channel code satisfies

$$
\mathbb{E}_{\mathcal{C}}(\epsilon)<\exp (-n \gamma)
$$

for some $\gamma>0$ and sufficiently large $n$.

With standard techniques, this theorem can be extended to the case of cost-constrained codebooks. We provide the full details of the proof of the following corollary in SectionVI-D

Corollary 1. In the setting of Theorem 3. and given an additive cost constraint $(c, C)$ compatible with $P$, there are $\gamma_{1}, \gamma_{2}>0$ such that for sufficiently large $n$,

$$
\mathbb{P}_{\mathcal{C}_{c, C}}\left(\epsilon \geq \exp \left(-n \gamma_{1}\right)\right)<\exp \left(-n \gamma_{2}\right)
$$

\section{ImplicATIONS OF THE MAIN RESUlts}

In this section, we show that the main result on secure OTA computation, Theorem 2, implies a MSE security guarantee in the case of AWGN channels discussed in Section III. To this end, we show in Section $\mathrm{V}-\mathrm{A}$ that AWGN compound channels satisfy (among other channel models) the approximability criterion of Definition 4 In Section V-B, we show how Theorem 2] can be applied to carry the MSE security result of Lemma 2 over to the case in which the legitimate receiver does not share randomness with the jammer.

\section{A. Feasibility of Channel Approximation}

In this subsection, we provide some tools and examples to argue that many compound channels of practical interest can indeed be $(\delta, J)$-approximated so that Theorem 3 may be applied to them. We begin with an observation that shows how our result specializes to the known results [12], [13] for channels with finite alphabets.

Remark 1. [12. Lemma 4] implies that for every compound channel $\left(W_{s}\right)_{s \in \mathcal{S}}$ with finite input and output alphabets and every $\delta>0$, there is an integer $J(\delta)$ such that $\left(W_{s}\right)_{s \in \mathcal{S}}$ can be $(\delta, J(\delta))$-approximated.

We repeat the construction here and discuss how this fact is proved. 
Let $M$ be an integer which satisfies

$$
M \geq \max \left(\frac{4|\mathcal{Y}|^{3}}{\delta^{2}}, \frac{2|\mathcal{Y}|^{2}}{\delta}\right) .
$$

Given $s \in \mathcal{S}$, we construct a channel $W_{s}^{\prime}$. To this end, given any $x \in \mathcal{X}$, we fix an enumeration $\left(y_{k}\right)_{k=1}^{|\mathcal{Y}|}$ such that the finite sequence $\left(W_{s}\left(x,\left\{y_{n}\right\}\right)\right)_{k=1}^{|\mathcal{Y}|}$ is nondecreasing. For every $k<|\mathcal{Y}|$, we can then uniquely choose a value for $W_{s}^{\prime}\left(x,\left\{y_{n}\right\}\right)$ such that it is an integer multiple of $1 / M$ and

$$
W_{s}\left(x,\left\{y_{n}\right\}\right) \leq W_{s}^{\prime}\left(x,\left\{y_{n}\right\}\right)<W_{s}\left(x,\left\{y_{n}\right\}\right)+\frac{1}{M}
$$

It is argued in [12] that this leaves a positive probability mass for $W_{s}^{\prime}\left(x,\left\{y_{|\mathcal{Y}|}\right\}\right)$ and therefore, this construction fully defines a channel $W_{s}^{\prime}$. We define the approximation sequence $\left(\hat{W}_{\delta, j}\right)_{j=1}^{J(\delta)}$ as an enumeration of the set $\left\{W_{s}^{\prime}: s \in \mathcal{S}\right\}$. The cardinality of this set is upper bounded by $(M+1)^{|\mathcal{X}||\mathcal{Y}|}$ since all singleton probabilities are integer multiples of $1 / M$.

For finite alphabets, (10) is trivially satisfied since Rényi divergence is in this case always finite [38]. Regarding the absolute continuity criterion $(11]$, we recall that $W_{s}^{\prime}\left(x,\left\{y_{|\mathcal{Y}|}\right\}\right)$ always has a positive probability, and for $k<|\mathcal{Y}|$, the assumption $W_{s}\left(x,\left\{y_{n}\right\}\right)=0$ immediately implies $W_{s}^{\prime}\left(x,\left\{y_{|\mathcal{Y}|}\right\}\right)=0$ by (20), since 0 is the only integer multiple of $1 / M$ which is strictly smaller than $1 / M$. The proof in [12] exploits (20) to prove that the absolute difference between the information of $W_{s}$ and $W_{s}^{\prime}$ under any input distribution is at most $2|\mathcal{Y}|^{3 / 2} M^{-1 / 2}$ (statement (c) of the lemma) which by our choice of $M$ immediately implies (12) and (13). Moreover, it is shown that (20) also implies that for all $x \in \mathcal{X}, y \in \mathcal{Y}$,

$$
\log \frac{W_{s}(x,\{y\})}{W_{s}^{\prime}(x,\{y\})} \leq \frac{2|\mathcal{Y}|^{2}}{M}
$$

(statement (b) of the lemma) which by our choice of $M$ implies (9).

For many channels of interest, $(\delta, J(\delta))$-approximability can be shown directly by going through properties (9) - 13. However, it is often easier to make an argument involving topological properties of $\mathcal{S}$. The following lemma provides some machinery to this end.

Lemma 5. Let $\left(W_{s}\right)_{s \in \mathcal{S}}$ be a compound channel with input alphabet $\mathcal{X}$ and output alphabet $\mathcal{Y}$, let $P$ be a probability distribution on $\mathcal{X}$ and assume that there is a topology on $\mathcal{S}$ such that $\mathcal{S}$ is compact and

$$
\begin{array}{r}
\forall s_{0} \in \mathcal{S}: s \mapsto \mathbb{E}_{P} \mathbf{D}_{1}\left(W_{s}(X, \cdot) \| W_{s_{0}}(X, \cdot)\right) \\
\text { is upper semi-continuous at } s_{0} \\
\forall s_{1}, s_{2} \in \mathcal{S} \exists \alpha>1 \forall x \in \mathcal{X}: \\
\quad \mathbf{D}_{\alpha}\left(W_{s_{1}}(x, \cdot) \| W_{s_{2}}(x, \cdot)\right)<\infty
\end{array}
$$

$s \mapsto \mathbf{I}_{P, W_{s}}$ is lower semi-continuous.

Then, for any $\delta>0$, there is $J(\delta)$ such that $\left(W_{s}\right)_{s \in \mathcal{S}}$ can be $(\delta, J(\delta))$-approximated under $P$.

Proof. Fix some $\delta>0$. For a given $s \in \mathcal{S}$, consider

$$
\begin{aligned}
\left\{s^{\prime}: \mathbb{E}_{P} \mathbf{D}_{1}\left(W_{s^{\prime}}(X, \cdot) \| W_{s}(X, \cdot)\right)<\delta\right\} & \\
& \cap\left\{s^{\prime}: \mathbf{I}_{P, W_{s}}-\mathbf{I}_{P, W_{s^{\prime}}}<\delta .\right\}
\end{aligned}
$$

Clearly, (21) and (23) ensure that this intersection is a neighborhood of $s$, so we can find an open neighborhood $\mathcal{A}_{s}$ contained in it. Thus, $\left(\mathcal{A}_{s}\right)_{s \in \mathcal{S}}$ is an open cover of $\mathcal{S}$ and therefore, the compactness of $\mathcal{S}$ yields a finite subcover $\mathcal{A}_{s_{1}}, \ldots, \mathcal{A}_{s_{J(\delta)}}$. We set $\hat{W}_{\delta, j}:=W_{s_{j}}$ and given any $s \in \mathcal{S}$, we choose $j$ such that $s \in \mathcal{A}_{s_{j}}$ and argue that $\hat{W}_{\delta, j}$ satisfies (9), (10) and (12). To this end, we note that (10) and (11) follow from (22), while (9) and (12) are ensured by the definition of $\mathcal{A}_{s_{j}}$. Finally, (13) is trivially satisfied, concluding the proof.

We now make use of Lemma 5 to prove that a large class of Gaussian fading multiple-input and multiple-output channels can actually be $(\delta, J(\delta))$-approximated and thus Theorem 3 can be applied to them. The class of compound channels covered in the following theorem contains the class considered in [8. Sections 3 and 4] as a proper subset. We denote the set of symmetric, positive semidefinite $n \times n$-matrices with $\mathrm{Sym}_{+}^{n}$ and the set of symmetric, positive definite $n \times n$-matrices with Sym $_{++}^{n}$.

Theorem 4. Let $\mathcal{X}=\mathbb{R}^{j}, \mathcal{Y}=\mathbb{R}^{i}$, let $\mathcal{S}$ be a compact subset of $\mathbb{R}^{i j} \times \mathrm{Sym}_{+}^{i j} \times \mathbb{R}^{i} \times \mathrm{Sym}_{++}^{i}$ (under the topology induced by the Frobenius norm). For any $s=\left(\mu_{H}, \Sigma_{H}, \mu_{N}, \Sigma_{N}\right) \in \mathcal{S}$, let $W_{s}$ be the channel given by

$$
Y=H X+N
$$

where the channel input $X$ has range $\mathbb{R}^{j}$, the channel output $Y$ has range $\mathbb{R}^{i}$, the entries of the $i \times j$ fading matrix $H$ follow the distribution $\mathcal{N}\left(\mu_{H}, \Sigma_{H}\right)$ and the additive noise $N$ is independent of $H$ and follows the distribution $\mathcal{N}\left(\mu_{N}, \Sigma_{N}\right)$. Let $P$ be a distribution on $\mathcal{X}$ and assume that either $P$ is a multivariate Gaussian with positive definite covariance matrix or that the support of $P$ is contained in some compact set. Then, given any $\delta>0$, there is $J(\delta)$ such that $\left(W_{s}\right)_{s \in \mathcal{S}}$ can be $(\delta, J(\delta))$-approximated under $P$.

Proof. We show that the conditions of Lemma 5] are met. [39] provides closed-form expressions for Rényi and KullbackLeibler divergences between multivariate normal distributions. The only fact that we are going to use and which is apparent from these expressions, however, is that the Rényi and Kullback-Leibler divergences between two multivariate normal distributions are finite and continuous in the mean vectors and covariance matrices of the distributions wherever the covariance matrices are positive definite or, equivalently, both distributions are absolutely continuous with respect to the Lebesgue measure.

$\Sigma_{N} \in \mathrm{Sym}_{++}^{i}$ and therefore, given any $x \in \mathcal{X}, W_{s}(x, \cdot)$ is absolutely continuous with respect to the Lebesgue measure and thus has a positive definite covariance matrix and a density $p_{W_{s}(x, \cdot)}$, which implies (22).

Next, from the well-known closed-form expression of the multivariate normal density, we know that for any $x$ and $y$, $p_{W_{s}(x, \cdot)}(y)$ is continuous in $s$. The boundedness of $\mathcal{S}$ implies a uniform upper bound on $p_{W_{s}(x, \cdot)}(y)$, so we can use the theorem of dominated convergence to argue that the marginal 
density $p_{R_{P, W_{s}}}(y)=\mathbb{E}_{P} p_{W_{s}(X, \cdot)}(y)$ depends continuously on $s$ for any fixed $y$. We write

$$
\mathbf{I}_{P, W_{s}}=\mathbb{E}_{P R_{P, W_{s}}}\left(\frac{p_{W_{s}(X, \cdot)}(Y)}{p_{R_{P, W_{s}}}(Y)} \log \frac{p_{W_{s}(X, \cdot)}(Y)}{p_{R_{P, W_{s}}}(Y)}\right) .
$$

Since the integrand is lower bounded by $-1 / e$, (23) follows as an application of Fatou's lemma.

Finally, in order to argue (21), we distinguish between the two cases in the statement of the theorem.

First, suppose that there is a compact subset $\hat{\mathcal{X}} \subseteq \mathcal{X}$ with $P(\mathcal{X} \backslash \hat{\mathcal{X}})=0$. For any fixed $s_{0}$, the map

$$
(s, x) \mapsto \mathbf{D}_{1}\left(W_{s}(x, \cdot) \| W_{s_{0}}(x, \cdot)\right)
$$

is continuous, therefore the image of $\mathcal{S} \times \hat{\mathcal{X}}$ is compact and hence bounded. We can therefore invoke the theorem of dominated convergence and argue that (21) is satisfied.

Now, suppose that $P$ is multivariate Gaussian with positive definite covariance matrix. We write

$$
\begin{aligned}
& \mathbb{E}_{P} \mathbf{D}_{1}\left(W_{s}(X, \cdot) \| W_{s_{0}}(X, \cdot)\right) \\
= & \mathbb{E}_{P} \mathbb{E}_{W_{s}(X, \cdot)} \log \frac{p_{P}(X) p_{W_{s}(X, \cdot)}(Y)}{p_{P}(X) p_{W_{s_{0}}(X, \cdot)}(Y)} \\
= & \mathbb{E}_{Q_{P, W_{s}}} \log \frac{p_{Q_{P, W_{s}}}(X, Y)}{p_{Q_{P, W_{s_{0}}}}(X, Y)} \\
= & \mathbf{D}_{1}\left(Q_{P, W_{s}} \| Q_{P, W_{s_{0}}}\right) .
\end{aligned}
$$

From our arguments above, given any $s$, the distribution $Q_{P, W_{s}}$ is multivariate Gaussian with positive definite covariance matrix, which implies that 21 is satisfied.

\section{B. Back to the AWGN case: Calculating MSE Security Guar- antees}

Revisiting the AWGN example from Section III, Theorem 2 and Lemma 2 imply MSE security and reconstruction guarantees in case the legitimate receiver does not know the jamming sequence, as we show in the following corollary.

Corollary 2. Make the same assumptions and definitions as in Lemma 2 but do not assume that the legitimate receiver has knowledge of the jamming sequence $X_{1}, \ldots, X_{n}$. Assume in addition that the channel from $\mathfrak{J}$ to $\mathfrak{B}$ is stronger than the channel from $\mathfrak{J}$ to $\mathfrak{E}$, i.e., $h_{\mathfrak{J} \mathfrak{B}} / \sigma_{\mathfrak{B}}>h_{\mathfrak{J E}} / \sigma_{\mathfrak{E}}$. Then there is a distributed approximation scheme with jamming and there are constants $\gamma_{1}, \gamma_{2}>0$ such that for sufficiently large $n$, the following hold:

- $\mathfrak{B}$ can approximate the objective function $f\left(s_{1}, \ldots, s_{K}\right)$ with a MSE not exceeding

$$
\sigma_{\text {eff }, \mathfrak{B}}^{2} \Psi\left(\frac{2}{\sigma_{\text {eff }, \mathfrak{B}}}\right)+\exp \left(-n \gamma_{1}\right)
$$

- The scheme is $(f, V)$-MSE-secure, where

$$
V:=\sigma_{\text {eff, }}^{2} \Psi\left(\frac{2}{\sigma_{\text {eff,eE }}}\right)-\exp \left(-n \gamma_{2}\right) .
$$

Proof. For the pre-processing at the transmitters, we use the same scheme as in the proof of Lemma 2 and begin by verifying that the resulting effective channels $W_{\mathfrak{B}}$ and $W_{\mathfrak{E}}$ with the input distribution $P$ chosen to be Gaussian with mean 0 and variance $\mathfrak{P}_{\mathfrak{J}}$ satisfy the assumptions of Theorem 2 . Since the defined compound channel is a class of Gaussian channels with different means taking values in the compact set $[-1,1]$, the approximability of the channel is an immediate consequence of Theorem 4 The finiteness of the momentgenerating function of the information density can be seen by straightforward applications of the definitions of information density and Rényi divergence:

$$
\begin{aligned}
& \mathbb{E} \exp \left(t \cdot \mathbf{i}_{P, W_{\mathfrak{E}}\left(s_{1}, \ldots, s_{K}, \cdot, \cdot\right)}(X ; Z)\right) \\
& =\mathbb{E}\left(\left(\frac{d W_{\mathfrak{E}}\left(s_{1}, \ldots, s_{K}, X, \cdot\right)}{d R_{P, W_{\mathfrak{E}}}\left(s_{1}, \ldots, s_{K}, \cdot, \cdot\right)}(Z)\right)^{t}\right) \\
& =\exp \left(t \cdot \frac{1}{t} \log \mathbb{E}\left(\left(\frac{d W_{\mathfrak{E}}\left(s_{1}, \ldots, s_{K}, X, \cdot\right)}{d R_{P, W_{\mathfrak{E}}}\left(s_{1}, \ldots, s_{K}, \cdot, \cdot\right)}(Z)\right)^{t}\right)\right) \\
& =\exp \left(t \mathbf{D}_{t+1}\left(Q_{P, W_{\mathfrak{E}}\left(s_{1}, \ldots, s_{K}, \cdot, \cdot\right)} \| P R_{P, W_{\mathfrak{E}}\left(s_{1}, \ldots, s_{K},, \cdot, \cdot\right)}\right)\right)
\end{aligned}
$$

The Rényi divergence appearing at the end is between two multivariate Gaussian distributions and can be seen to be finite from the expressions given in [39]. In order to verify (14), we first note that the information expressions appearing are the capacities of the effective channels $W_{\mathfrak{B}}$ and $W_{\mathfrak{E}}$. Since $s_{1}, \ldots, s_{K}$ change the mean of the channel only, they do not influence the capacity. Therefore, the infimum and supremum are over singleton sets. Consequently, the condition $h_{\mathfrak{J} \mathfrak{B}} / \sigma_{\mathfrak{B}}>h_{\mathfrak{J} \mathfrak{E}} / \sigma_{\mathfrak{E}}$ ensures that there is some $\mathcal{R}$ satisfying (14).

Fix $\gamma_{1}{ }^{\prime}, \gamma_{3}{ }^{\prime}$ as claimed to exist in Theorem 2, and also fix $\gamma_{1}, \gamma_{2}$ with $0<\gamma_{2}<\gamma_{1}{ }^{\prime}$ and $0<\gamma_{1}<\gamma_{3}{ }^{\prime}$.

Note that in the AWGN channel, $s_{1}, \ldots, s_{K}$ correspond to a shift of the output distribution of the channel, and therefore, the variational distance that appears in (15) is independent of $s_{1}, \ldots, s_{K}$. For sufficiently large $n$, we can therefore fix a codebook $\mathcal{C}$ from the $(P, n, \mathcal{R})$-ensemble such that for all $s_{1}, \ldots, s_{K}$, neither one of the error events described in (15) and (16) occurs.

Let the jamming strategy be induced by $\mathcal{C}_{C, c}$ and let $d$ : $\mathcal{Z}^{n} \rightarrow[-1,1]$ be an estimator for $\mathfrak{E}$. We can now bound the MSE of $d$ :

$$
\begin{gathered}
\mathbb{E}_{\hat{R}_{W_{\mathfrak{E}}\left(s_{1}, \ldots, s_{K},, \cdot,\right)^{n}}}\left(\left(d\left(Z^{n}\right)-f\left(s_{1}, \ldots, s_{K}\right)\right)^{2}\right) \\
=\int_{0}^{4} \hat{R}_{W_{\mathfrak{E}}\left(s_{1}, \ldots, s_{K}, \cdot, \cdot\right)^{n}}\left(\left(d\left(Z^{n}\right)-f\left(s_{1}, \ldots, s_{K}\right)\right)^{2}>t\right) d t \\
\frac{15}{\geq} \int_{0}^{4}\left(R_{P, W_{\mathfrak{E}}\left(s_{1}, \ldots, s_{K},, \cdot,\right)}^{n}\left(\left(d\left(Z^{n}\right)-f\left(s_{1}, \ldots, s_{K}\right)\right)^{2}>t\right)\right. \\
\left.-\exp \left(-n \gamma_{1}{ }^{\prime}\right)\right) d t \\
=\mathbb{E}_{R_{P, W_{\mathfrak{E}}\left(s_{1}, \ldots, s_{K}, \cdot, \cdot\right)}^{n}\left(\left(d\left(Z^{n}\right)-f\left(s_{1}, \ldots, s_{K}\right)\right)^{2}\right)}-4 \exp \left(-n \gamma_{1}{ }^{\prime}\right) .
\end{gathered}
$$

Taking the lower bound for the MSE under $R_{P, W_{\mathfrak{E}}\left(s_{1}, \ldots, s_{K},, \text {. }\right)}^{n}$ from Lemma 2 and noting $\gamma_{2}<\gamma_{1}{ }^{\prime}$, we arrive at the expression in (25) for sufficiently large $n$.

For the reconstruction strategy at $\mathfrak{B}$, we first let $\mathfrak{B}$ reconstruct the jamming signal as is possible by Theorem 2 and then post-process the received signal as is possible with knowledge 
of the jamming signal by Lemma 2 Using the error bound in Lemma 2 and observing that the maximum instantaneous square error is 4 since we are constrained to an interval of length 2 and that $\gamma_{1}<\gamma_{3}{ }^{\prime}$, for sufficiently large $n$ we arrive at (24).

\section{PROOFS}

In this section, we prove the Lemmas used in the proof of Lemma 2 and our main results on secure OTA computation and compound channel coding, Theorems 2 and 3 , as well as the corollaries that allow for the incorporation of an average cost constraint.

\section{A. Statistical Preliminaries for the Proof of Lemma 2}

In this subsection, we prove the two lemmas used for the proof of Lemma 2

Proof of Lemma 3 It is known [40, eq. (6.92)] that the MSE is minimized by the mean of the posterior probability distribution. We can therefore calculate the minimum MSE estimator given the observations $v_{1}, \ldots, v_{n}$ as follows, where we use $p$ with random variables in the index to denote (conditional) densities.

$$
\begin{aligned}
\hat{U} & =\int_{a}^{b} u p_{U \mid V_{1}, \ldots, V_{n}}\left(u \mid v_{1}, \ldots, v_{n}\right) d u \\
& \stackrel{(a)}{=} \int_{a}^{b} u \frac{p_{V_{1}, \ldots, V_{n} \mid U}\left(v, \ldots, v_{n} \mid u\right) p_{U}(u)}{p_{V_{1}, \ldots, V_{n}}\left(v_{1}, \ldots, v_{n}\right)} d u \\
& =\frac{\int_{a}^{b} u p_{V_{1}, \ldots, V_{n} \mid U}\left(v_{1}, \ldots, v_{n} \mid u\right) p_{U}(u) d u}{\int_{a}^{b} p_{V_{1}, \ldots, V_{n} \mid U}\left(v_{1}, \ldots, v_{n} \mid u\right) p_{U}(u) d u} \\
& \stackrel{(b)}{=} \frac{\int_{a}^{b} u \exp \left(-\frac{1}{2 \sigma^{2}} \sum_{j=1}^{n}\left(v_{j}-u\right)^{2}\right) d u}{\int_{a}^{b} \exp \left(-\frac{1}{2 \sigma^{2}} \sum_{j=1}^{n}\left(v_{j}-u\right)^{2}\right) d u} \\
& =\frac{\int_{a}^{b} u \exp \left(-\frac{1}{2 \sigma^{2} / n}\left(\frac{1}{n} \sum_{j=1}^{n} v_{j}^{2}-2 u \bar{v}+u^{2}\right)\right) d u}{\int_{a}^{b} \exp \left(-\frac{1}{2 \sigma^{2} / n}\left(\frac{1}{n} \sum_{j=1}^{n} v_{j}^{2}-2 u \bar{v}+u^{2}\right)\right) d u} \\
& \stackrel{(c)}{=} \frac{\int_{a}^{b} u \exp \left(-\frac{1}{2 \sigma^{2} / n}(\bar{v}-u)^{2}\right) d u}{\int_{a}^{b} \exp \left(-\frac{1}{2 \sigma^{2} / n}(\bar{v}-u)^{2}\right) d u}
\end{aligned}
$$

For (a), we have applied Bayes' rule. (b) is by observing that $p_{U}(u)=1 /(b-a)$ is independent of $u$ in $[a, b]$ and $p_{V_{1}, \ldots, V_{n} \mid U}$ is the normal density. (c) is by multiplying

$$
\exp \left(-\frac{1}{2 \sigma^{2} / n}\left(\bar{v}^{2}-\frac{1}{n} \sum_{j=1}^{n} v_{j}^{2}\right)\right)
$$

on both sides of the fraction to complete the binomials.

The term we have calculated for $\hat{U}$ is the mean of a normal distribution centered at $\bar{v}$ with variance $\sigma^{2} / n$ truncated in $[a, b]$. This is a distribution with a known mean [41, eq. 13.134], and hence we arrive at (6).

Proof of Lemma 4 Based on the representation (6), we calculate the MSE as follows. We use the substitution rule, substituting $v^{\prime}:=\frac{\bar{v}-a}{\sigma / \sqrt{n}}$ in (a) and $u^{\prime}:=\frac{u-a}{\sigma / \sqrt{n}}$ in (b).

$$
\begin{aligned}
& \mathbb{E}\left((U-\hat{U})^{2}\right) \\
& =\int_{a}^{b} \int_{-\infty}^{\infty}\left(\bar{v}+\frac{\sigma}{\sqrt{n}} \cdot \frac{\varphi_{N}\left(\frac{a-\bar{v}}{\sigma / \sqrt{n}}\right)-\varphi_{N}\left(\frac{b-\bar{v}}{\sigma / \sqrt{n}}\right)}{\Phi_{N}\left(\frac{b-\bar{v}}{\sigma / \sqrt{n}}\right)-\Phi_{N}\left(\frac{a-\bar{v}}{\sigma / \sqrt{n}}\right)}-u\right)^{2} \\
& \frac{1}{b-a} \cdot \frac{1}{\sigma / \sqrt{n}} \varphi_{N}\left(\frac{u-\bar{v}}{\sigma / \sqrt{n}}\right) d \bar{v} d u \\
& \stackrel{(a)}{=} \int_{a}^{b} \int_{-\infty}^{\infty}\left(\frac{\sigma}{\sqrt{n}}\left(v^{\prime}+\frac{\varphi_{N}\left(-v^{\prime}\right)-\varphi_{N}\left(\frac{b-a}{\sigma / \sqrt{n}}-v^{\prime}\right)}{\Phi_{N}\left(\frac{b-a}{\sigma / \sqrt{n}}-v^{\prime}\right)-\Phi_{N}\left(-v^{\prime}\right)}\right)\right. \\
& +a-u)^{2} \cdot \frac{1}{b-a} \cdot \varphi_{N}\left(\frac{u-a}{\sigma / \sqrt{n}}-v^{\prime}\right) d v^{\prime} d u \\
& \stackrel{(b)}{=} \int_{0}^{\frac{b-a}{\sigma / \sqrt{n}}} \int_{-\infty}^{\infty}\left(v^{\prime}+\frac{\varphi_{N}\left(-v^{\prime}\right)-\varphi_{N}\left(\frac{b-a}{\sigma / \sqrt{n}}-v^{\prime}\right)}{\Phi_{N}\left(\frac{b-a}{\sigma / \sqrt{n}}-v^{\prime}\right)-\Phi_{N}\left(-v^{\prime}\right)}-u^{\prime}\right)^{2} \\
& \cdot\left(\frac{\sigma}{\sqrt{n}}\right)^{3} \cdot \frac{1}{b-a} \cdot \varphi_{N}\left(u^{\prime}-v^{\prime}\right) d v^{\prime} d u^{\prime} \\
& =\frac{\sigma^{2}}{n} \Psi\left(\frac{b-a}{\sigma / \sqrt{n}}\right),
\end{aligned}
$$

concluding the proof of the lemma.

\section{B. Proof of Theorem 2}

In order to prove Theorem 2, we decompose the system depicted in Fig. 4 into smaller (and more easily analyzed) subsystems by considering only a subset of the depicted terminals at a time.

a) Considering the terminals $\mathfrak{A}_{1}, \ldots, \mathfrak{A}_{K}, \mathfrak{B}$ : This is the system summarized in Section [I-A. The rationale is that the results specialize to the setting in Section $\amalg$ as well as, e.g., to the fast-fading setting introduced in [4]. This part of the system consists of transmitters $\left(\mathfrak{A}_{k}\right)_{k=1}^{K}$ each of which holds a value $s_{k} \in \mathcal{S}_{k}$ and a receiver $\mathfrak{B}$ which has the objective of estimating $f\left(s_{1}, \ldots, s_{K}\right)$. To this end, each transmitter $\mathfrak{A}_{k}$ passes $s_{k}$ through a pre-processor $F_{k}$ independently $n$ times yielding a sequence $T_{k}^{n}$ of channel inputs. These are transmitted through $n$ independent uses of the channel, generating a sequence $Y^{n}$ of channel outputs. The receiver passes this sequence through a post-processor $D^{n}$ which generates an approximation $\tilde{f}$ of $f\left(s_{1}, \ldots, s_{K}\right)$. As mentioned, the design of the pre- and post-processors depends heavily on the channel model and a particular class of functions $f$. The idea is that the preprocessors, the channel and the post-processor work together to mimic the function $f$, and any approach following this idea will be highly dependent on the particular structure of the channel and $f$. In Theorem 2, it is assumed that such a system is already in place and an augmentation is proposed which makes it more secure. A property of the system described in Section $\amalg-A$ necessary for our purposes and heavily exploited in this work is that the pre-processing is i.i.d., i.e., each preprocessor $F_{k}$ is a stochastic kernel mapping from $\mathcal{S}_{k}$ to $\mathcal{T}_{k}$ 
and an $n$-fold product $F_{k}^{n}$ of it is used to generate the channel input sequence.

b) Considering the terminals $\mathfrak{A}_{1}, \ldots, \mathfrak{A}_{K}, \mathfrak{J}, \mathfrak{E}:$ In this setting, we assume that the transmitters $\mathfrak{A}_{1}, \ldots, \mathfrak{A}_{K}$ run a scheme of the kind described undera). Instead of the legitimate receiver, there is now an eavesdropper $\mathfrak{E}$. The objective is then to limit the usefulness of the eavesdropper's received signal $Z^{n}$. To this end, we add a friendly jammer $\mathfrak{J}$ to the system which transmits, according to a certain strategy, a word $X^{n}$. In this work, any jamming strategy we consider is induced by a codebook $\mathcal{C}$ of words of length $n$ through the rule that the jammer chooses an element of the codebook uniformly at random and transmits it. We use existing results on channel resolvability to derive a bound on the usefulness of the signal $Z^{n}$ received at $\mathfrak{E}$.

c) Considering the terminals $\mathfrak{A}_{1}, \ldots, \mathfrak{A}_{K}, \mathfrak{J}, \mathfrak{B}:$ This is the setting from (a) with an additional transmitter $\mathfrak{J}$. Here we assume that $\mathfrak{J}$ uses a jamming strategy induced by a codebook $\mathcal{C}$ as described under $b$ and use Theorem 3 on compound channel coding to argue that for suitable choices of $\mathcal{C}, \mathfrak{B}$ is able to fully reconstruct the jamming signal $X^{n}$. This enables $\mathfrak{B}$ to perform a cancellation of the jamming signal before it applies the post-processor $D^{n}$ it would use in setting a). How this cancellation works depends on the particularities of the channel considered, but if, e.g., the jamming signal is simply added to the channel output as in the AWGN example in Section III it is possible to cancel it entirely by subtracting it from the received signal. So in this case the post-processor would consist of a reconstruction of the jamming signal, the subtraction of this signal from the received one and a postprocessing step identical to that from a).

d) Combining settings $(b$ and $(c)$ : The goal here is to argue the existence of a $\operatorname{codebook} \mathcal{C}$ which achieves both of the objectives described under $\mathrm{b}$ and (c). It will turn out that this can be achieved by a standard random codebook construction.

The main result of this work, Theorem 2 , formulates conditions under which there are codebooks in the $(P, n, \mathcal{R})$ ensemble of which the $(c, C)$-cost constrained versions simultaneously achieve the goals set forth under b) and c).

As a technical ingredient for our proof, we recall a result on channel resolvability from [23] that will be applied in order to guarantee the virtual indistinguishability of the jamming signal from white noise for the eavesdropper.

Theorem 5. [23] Given a channel $W$ from $\mathcal{X}$ to $\mathcal{Y}$, an input distribution $P$ such that the moment-generating function $\mathbb{E}_{Q_{P, W}} \exp \left(t \cdot \mathbf{i}_{P, W}(X ; Y)\right)$ of the information density exists and is finite for some $t>0$, and $\mathcal{R}>\mathbf{I}_{P, W}$, there exist $\gamma_{1}>0$ and $\gamma_{2}>0$ such that for large enough block lengths $n$, the $(P, n, \mathcal{R})$-ensemble satisfies

$$
\begin{aligned}
\mathbb{P}_{\mathcal{C}}\left(\left\|\hat{R}_{W, \mathcal{C}}-Q_{P, W}^{n}\right\|_{\mathrm{TV}}>\exp \left(-\gamma_{1} n\right)\right) \\
\leq \exp \left(-\exp \left(\gamma_{2} n\right)\right),
\end{aligned}
$$

where $\hat{R}_{W, \mathcal{C}}$ is the output distribution of channel $W$ given that a uniformly random codeword from $\mathcal{C}$ is transmitted.

Similarly as with the compound channel coding theorem, we can use known methods to incorporate an additive cost constraint and argue the following corollary. For full details, we refer the reader to Section VI-D

Corollary 3. Let $P$ be an input distribution on $\mathcal{X}$ and $(c, C)$ an additive cost constraint compatible with $P$. Then the statement of Theorem 5 is valid even if the codebook $\mathcal{C}$ is replaced with its associated cost-constrained version $\mathcal{C}_{c, C}$.

Given the previous observations, the proof of the main result of this work is now straightforward.

Proof of Theorem 2 An application of Corollary 11 yields (16), and (15) follows from Corollary 3

\section{Proof of Theorem 3}

We first pick parameters $\delta, \varepsilon, \beta_{1}$ and $\beta_{2}$ in sequence according to the following scheme, where (17) and the previous choices ensure that these intervals are all nonempty.

$$
\begin{aligned}
\delta & \in\left(0, \frac{\inf _{s \in \mathcal{S}} \mathbf{I}_{P, W_{s}}-\mathcal{R}}{3}\right) \\
\varepsilon & \in\left(2 \delta \inf _{s \in \mathcal{S}} \mathbf{I}_{P, W_{s}}-\mathcal{R}-\delta\right) \\
\beta_{1} & \in(\delta, \varepsilon-\delta) \\
\beta_{2} & \in\left(0, \varepsilon-\delta-\beta_{1}\right)
\end{aligned}
$$

Fix a sequence $\left(\hat{W}_{\delta, j}\right)_{j=1}^{J(\delta)}$ which $(\delta, J(\delta))$-approximates $\left(W_{s}\right)_{s \in \mathcal{S}}$

We use a joint typicality decoder, i.e. if there is a unique $m$ such that

$$
\exists j \in\{1, \ldots, J(\delta)\}: \mathbf{i}_{P, \hat{W}_{\delta, j}}\left(\mathcal{C}(m) ; Y^{n}\right) \geq n\left(\mathbf{I}_{P, \hat{W}_{\delta, j}}-\varepsilon\right),
$$

the decoder declares that message $m$ has been sent; otherwise it declares an error (or that message 1 has been sent).

We denote the transmitted message with $\mathcal{M}$, the message declared by the decoder with $\hat{\mathcal{M}}$ and define error events

$$
\begin{aligned}
& \mathcal{E}:=\{\mathcal{M} \neq \hat{\mathcal{M}}\} \\
& \mathcal{E}_{1}:=\{\forall j \in\{1, \ldots, J(\delta)\} \\
&\left.\mathbf{i}_{P, \hat{W}_{\delta, j}}\left(\mathcal{C}(\mathcal{M}) ; Y^{n}\right)<n\left(\mathbf{I}_{P, \hat{W}_{\delta, j}}-\varepsilon\right)\right\} \\
& \mathcal{E}_{2}:=\{\exists m \neq \mathcal{M} \exists j \in\{1, \ldots, J(\delta)\} \\
&\left.\quad \mathbf{i}_{P, \hat{W}_{\delta, j}}\left(\mathcal{C}(m) ; Y^{n}\right) \geq n\left(\mathbf{I}_{P, \hat{W}_{\delta, j}}-\varepsilon\right)\right\} .
\end{aligned}
$$

We note that $\mathcal{E} \subseteq \mathcal{E}_{1} \cup \mathcal{E}_{2}$ and consequently

$$
\mathbb{P}(\mathcal{E}) \leq \mathbb{P}\left(\mathcal{E}_{1}\right)+\mathbb{P}\left(\mathcal{E}_{2}\right)
$$

So we can bound these two errors separately and then combine them.

We start with bounding the expectation of the first summand, using the definition (32) and $\mathcal{C}$, as well as an addition 
of zero. Pick $j$ such that $\hat{W}_{\delta, j}$ satisfies (9) - (12) with respect to the realization $W_{s}$ of the compound channel. Then we have

$$
\begin{aligned}
& \mathbb{E}_{\mathcal{C}}\left(\mathbb{P}\left(\mathcal{E}_{1}\right)\right) \\
\leq & \mathbb{E}_{\mathcal{C}}\left(\mathbb{P}\left(\mathbf{i}_{P, \hat{W}_{\delta, j}}\left(\mathcal{C}(\mathcal{M}) ; Y^{n}\right)<n\left(\mathbf{I}_{P, \hat{W}_{\delta, j}}-\varepsilon\right)\right)\right) \\
= & Q_{P, W_{s}}^{n}\left(\mathbf{i}_{P, \hat{W}_{\delta, j}}\left(X^{n} ; Y^{n}\right)<n\left(\mathbf{I}_{P, \hat{W}_{\delta, j}}-\varepsilon\right)\right) \\
= & Q_{P, W_{s}}^{n}\left(\sum_{i=1}^{n} \log \left(\frac{d \hat{W}_{\delta, j}\left(X_{i}, \cdot\right)}{d R_{P, \hat{W}_{\delta, j}}}\left(Y_{i}\right)\right)\right. \\
& \left.\quad n\left(\mathbf{I}_{P, \hat{W}_{\delta, j}}+\mathbf{I}_{P, W_{s}}-\mathbf{I}_{P, W_{s}}-\varepsilon\right)\right)
\end{aligned}
$$

The Radon-Nikodym derivative can be split as

$$
\frac{d \hat{W}_{\delta, j}\left(X_{i}, \cdot\right)}{d R_{P, \hat{W}_{\delta, j}}}=\frac{d \hat{W}_{\delta, j}\left(X_{i}, \cdot\right)}{d W_{s}\left(X_{i}, \cdot\right)} \cdot \frac{d R_{P, W_{s}}}{d R_{P, \hat{W}_{\delta, j}}} \cdot \frac{d W_{s}\left(X_{i}, \cdot\right)}{d R_{P, W_{s}}} .
$$

This is possible because $\hat{W}_{\delta, j}(x, \cdot) \ll W_{s}(x, \cdot)$ by (11), $R_{P, W_{s}} \ll R_{P, \hat{W}_{\delta, j}}$ by $(9)$ and the joint convexity of KullbackLeibler divergence in its arguments, and $W_{s}(x, \cdot) \ll R_{P, W_{s}}$ for $P$-almost all $x$ by the properties of the marginalization.

We next bound tail probabilities corresponding to the three factors in (36) separately, starting with the first. To this end, we introduce a number $\alpha_{1}>1$ and argue, using Markov's inequality and the definition of Rényi divergence, that

$$
\begin{aligned}
& Q_{P, W_{s}}^{n}\left(\sum_{i=1}^{n} \log \frac{d W_{s}\left(X_{i}, \cdot\right)}{d \hat{W}_{\delta, j}\left(X_{i}, \cdot\right)}\left(Y_{i}\right) \geq n \beta_{1}\right) \\
&= Q_{P, W_{s}}^{n}\left(\exp \left(\left(\alpha_{1}-1\right) \sum_{i=1}^{n} \log \frac{d W_{s}\left(X_{i}, \cdot\right)}{d \hat{W}_{\delta, j}\left(X_{i}, \cdot\right)}\left(Y_{i}\right)\right)\right. \\
&\left.\geq \exp \left(\left(\alpha_{1}-1\right) n \beta_{1}\right)\right) \\
& \leq \mathbb{E}_{Q_{P, W_{s}}^{n}}\left(\left(\prod_{i=1}^{n}\left(\frac{d W_{s}\left(X_{i}, \cdot\right)}{d \hat{W}_{\delta, j}\left(X_{i}, \cdot\right)}\left(Y_{i}\right)\right)^{\alpha_{1}-1}\right)\right) \\
& \cdot \exp \left(-\left(\alpha_{1}-1\right) n \beta_{1}\right) \\
&=\exp \left(\sum_{i=1}^{n} \log \left(\mathbb{E}_{Q_{P, W_{s}}^{n}}\left(\left(\frac{d W_{s}\left(X_{i}, \cdot\right)}{d \hat{W}_{\delta, j}\left(X_{i}, \cdot\right)}\right)\right)\right)\right. \\
&\left.\cdot \exp \left(-\left(\alpha_{1}-1\right) n \beta_{1}\right)\right) \\
&=\exp \left(-\left(\alpha_{1}-1\right) n\right. \\
& \cdot\left(\beta_{1}-\mathbb{E}_{P} \mathbf{D}_{\alpha_{1}}\left(W_{s}(X, \cdot) \| \hat{W}_{\delta, j}(X, \cdot)\right)\right) .
\end{aligned}
$$

For the second factor, we argue in an analogous way, but using $\alpha_{2}>0$.

$$
\begin{aligned}
& R_{P, W_{s}}^{n}\left(\sum_{i=1}^{n} \log \frac{d R_{P, \hat{W}_{\delta, j}}}{d R_{P, W_{s}}}\left(Y_{i}\right) \geq n \beta_{2}\right) \\
&= R_{P, W_{s}}^{n}\left(\exp \left(\alpha_{2} \sum_{i=1}^{n} \log \frac{d R_{P, \hat{W}_{\delta, j}}}{d R_{P, W_{s}}}\left(Y_{i}\right)\right)\right. \\
&\left.\geq \exp \left(\alpha_{2} n \beta_{2}\right)\right) \\
& \leq \mathbb{E}_{R_{P, W_{s}}^{n}}\left(\prod_{i=1}^{n}\left(\frac{d R_{P, \hat{W}_{\delta, j}}}{d R_{P, W_{s}}}\left(Y_{i}\right)\right)^{\alpha_{2}}\right) \exp \left(-\alpha_{2} n \beta_{2}\right) \\
&= \exp \left(\left(\alpha_{2}-1\right) n \mathbf{D}_{\alpha_{2}}\left(R_{P, \hat{W}_{\delta, j}} \| R_{P, W_{s}}\right)-\alpha_{2} n \beta_{2}\right) .
\end{aligned}
$$

Finally, for the third factor, we use $\alpha_{3}<1$.

$$
\begin{gathered}
Q_{P, W_{s}}^{n}\left(\mathbf{i}_{P, W_{s}}\left(X^{n} ; Y^{n}\right)\right. \\
\left.<n\left(\mathbf{I}_{P, W_{s}}-\varepsilon+\beta_{1}+\beta_{2}+\delta\right)\right) \\
=Q_{P, W_{s}}^{n}\left(\exp \left(\left(\alpha_{3}-1\right) \mathbf{i}_{P, W_{s}}\left(X^{n} ; Y^{n}\right)\right)\right. \\
\left.>\exp \left(\left(\alpha_{3}-1\right) n\left(\mathbf{I}_{P, W_{s}}-\varepsilon+\beta_{1}+\beta_{2}+\delta\right)\right)\right) \\
\leq \mathbb{E}_{Q_{P, W_{s}}^{n}}\left(\prod_{i=1}^{n}\left(\frac{d W_{s}\left(X_{i}, \cdot\right)}{d R_{P, W_{s}}}\left(Y_{i}\right)\right)^{\alpha_{3}-1}\right) \\
\cdot \exp \left(-\left(\alpha_{3}-1\right) n\left(\mathbf{I}_{P, W_{s}}-\varepsilon+\beta_{1}+\beta_{2}+\delta\right)\right) \\
=\exp \left(-\left(1-\alpha_{3}\right) n\left(\mathbf{D}_{\alpha_{3}}\left(Q_{P, W_{s}} \| P R_{P, W_{s}}\right)\right.\right. \\
\left.\left.+\varepsilon-\mathbf{I}_{P, W_{s}}-\beta_{1}-\beta_{2}-\delta\right)\right),
\end{gathered}
$$

Clearly, by (36), the union bound and (12), (35) is upper bounded by the sum of (37), (38) and (39). Next, we argue that these expressions all vanish exponentially with $n \rightarrow \infty$, using the continuity of Rényi divergence in the order which is shown in [38, Theorem 7].

From (10), the theorem of monotone convergence and (9), we can conclude that

$$
\begin{aligned}
\lim _{\alpha_{1} \searrow 1} \mathbb{E}_{P} \mathbf{D}_{\alpha_{1}}\left(W_{s}\left(X_{i}, \cdot\right) \| \hat{W}_{\delta, j}\left(X_{i}, \cdot\right)\right) \\
\quad=\mathbb{E}_{P} \mathbf{D}_{1}\left(W_{s}\left(X_{i}, \cdot\right) \| \hat{W}_{\delta, j}\left(X_{i}, \cdot\right)\right) \leq \delta,
\end{aligned}
$$

so, 29) allows us to fix $\alpha_{1}$ at a value greater than 1 such that $\beta_{1}-\mathbb{E}_{P} \mathbf{D}_{\alpha_{1}}\left(W_{s}\left(X_{i}, \cdot\right) \| \hat{W}_{\delta, j}\left(X_{i}, \cdot\right)\right)>0$ and hence, (37) vanishes exponentially.

(38) is true for all $\alpha_{2}<1$. Since the inequalities are not strict, we can take the limit $\alpha_{2} \nearrow 1$ and argue that the statement is also valid for $\alpha_{2}=1$.

$\mathbf{D}_{\alpha_{3}}\left(Q_{P, W_{s}} \| P R_{P, W_{s}}\right)$ converges to $\mathbf{I}_{P, W_{s}}$ from below for $\alpha_{3} \nearrow 1$ and so (30) allows us to fix $\alpha_{3}$ at a value less than 1 such that $\mathbf{D}_{\alpha_{3}}\left(Q_{P, W_{s}} \| P R_{P, W_{s}}\right)+\varepsilon-\mathbf{I}_{P, W_{s}}-\beta_{1}-\beta_{2}-\delta>0$ and therefore, (39) also vanishes exponentially.

For the second summand in (34), we use the definition (33) to argue that $\mathbb{E}_{\mathcal{C}}\left(\mathbb{P}\left(\mathcal{E}_{2}\right)\right)$ is upper bounded by 


$$
\begin{aligned}
\exp (n \mathcal{R}) \sum_{j=1}^{J(\delta)} P^{n} R_{P, W_{s}}^{n}\left(\mathbf{i}_{P, \hat{W}_{\delta, j}}\left(X^{n} ; Y^{n}\right)\right. & \\
& \left.\geq n\left(\mathbf{I}_{P, \hat{W}_{\delta, j}}-\varepsilon\right)\right) .
\end{aligned}
$$

We define the indicator function

$$
\operatorname{ind}\left(x^{n}, y^{n}\right):= \begin{cases}1, & \mathbf{i}_{P, \hat{W}_{\delta, j}}\left(x^{n} ; y^{n}\right) \geq n\left(\mathbf{I}_{P, \hat{W}_{\delta, j}}-\varepsilon\right) \\ 0, & \text { otherwise. }\end{cases}
$$

Using the definition of information density for a change of measure and multiplying one, we rewrite the probability that appears in (40) as

$$
\begin{aligned}
& P^{n} R_{P, W_{s}}^{n}\left(\mathbf{i}_{P, \hat{W}_{\delta, j}}\left(X^{n} ; Y^{n}\right) \geq n\left(\mathbf{I}_{P, \hat{W}_{\delta, j}}-\varepsilon\right)\right) \\
= & \int_{\mathcal{X}^{n} \times \mathcal{Y}^{n}} \operatorname{ind}\left(x^{n}, y^{n}\right) \cdot P^{n} R_{P, W_{s}}^{n}\left(d x^{n}, d y^{n}\right) \\
= & \int_{\mathcal{X}^{n} \times \mathcal{Y}^{n}} \exp \left(-\mathbf{i}_{P, W_{s}}\left(x^{n} ; y^{n}\right)\right) \operatorname{ind}\left(x^{n}, y^{n}\right) Q_{P, W_{s}}^{n}\left(d x^{n}, d y^{n}\right) \\
= & \int_{\mathcal{X}^{n} \times \mathcal{Y}^{n}} \exp \left(-\mathbf{i}_{P, W_{s}}\left(x^{n} ; y^{n}\right)+\mathbf{i}_{P, \hat{W}_{\delta, j}}\left(x^{n} ; y^{n}\right)\right. \\
& \left.-\mathbf{i}_{P, \hat{W}_{\delta, j}}\left(x^{n} ; y^{n}\right)\right) \\
& \cdot \operatorname{ind}\left(x^{n}, y^{n}\right) Q_{P, W_{s}}^{n}\left(d x^{n}, d y^{n}\right)
\end{aligned}
$$

Because of the presence of the indicator, we can uniformly bound

$$
\mathbf{i}_{P, \hat{W}_{\delta, j}}\left(x^{n} ; y^{n}\right) \geq n\left(\mathbf{I}_{P, \hat{W}_{\delta, j}}-\varepsilon\right)
$$

and the indicator itself can be upper bounded by 1 . This yields

$$
\begin{aligned}
& P^{n} R_{P, W_{s}}^{n}\left(\mathbf{i}_{P, \hat{W}_{\delta, j}}\left(X^{n} ; Y^{n}\right) \geq n\left(\mathbf{I}_{P, \hat{W}_{\delta, j}}-\varepsilon\right)\right) \\
& \leq \exp \left(-n\left(\mathbf{I}_{P, \hat{W}_{\delta, j}}-\varepsilon\right)\right) \\
& \int_{\mathcal{X}^{n} \times \mathcal{Y}^{n}} \exp \left(-\mathbf{i}_{P, W_{s}}\left(x^{n} ; y^{n}\right)+\mathbf{i}_{P, \hat{W}_{\delta, j}}\left(x^{n} ; y^{n}\right)\right) \\
& \cdot Q_{P, W_{s}}^{n}\left(d x^{n}, d y^{n}\right) .
\end{aligned}
$$

We expand the definition of information density and apply Fubini's Theorem to rewrite the integral as

$$
\int_{\mathcal{Y}^{n}}\left(\int_{\mathcal{X}^{n}} \frac{d \hat{W}_{\delta, j}^{n}\left(x^{n}, \cdot\right)}{d R_{P, \hat{W}_{\delta, j}^{n}}^{n}}\left(y^{n}\right) P^{n}\left(d x^{n}\right)\right) R_{P, W_{s}}^{n}\left(d y^{n}\right)
$$

and observe that it equals 1 .

Combining with (40) and applying (13), we obtain

$$
\begin{aligned}
& \mathbb{E}_{\mathcal{C}}\left(\mathbb{P}\left(\mathcal{E}_{2}\right)\right) \\
\leq & \exp (n \mathcal{R}) \sum_{j=1}^{J(\delta)} \exp \left(-n\left(\mathbf{I}_{P, \hat{W}_{\delta, j}}-\varepsilon\right)\right) \\
\leq & \exp (n \mathcal{R}) \sum_{j=1}^{J(\delta)} \exp \left(-n\left(\inf _{s \in \mathcal{S}} \mathbf{I}_{P, W_{s}}-\varepsilon-\delta\right)\right) \\
= & \exp \left(-n\left(\inf _{s \in \mathcal{S}} \mathbf{I}_{P, W_{s}}-\varepsilon-\mathcal{R}-\delta-\frac{\log J(\delta)}{n}\right)\right) .
\end{aligned}
$$

We observe that by (28), $\inf _{s \in \mathcal{S}} \mathbf{I}_{P, W_{s}}-\varepsilon-\mathcal{R}-\delta>0$. Finally, we pick

$$
\begin{aligned}
\gamma \in(0, \min ( & \left(\alpha_{1}-1\right) \\
& \cdot\left(\beta_{1}-\mathbb{E}_{P} \mathbf{D}_{\alpha_{1}}\left(W_{s}\left(X_{i}, \cdot\right) \| \hat{W}_{\delta, j}\left(X_{i}, \cdot\right)\right)\right), \\
& \beta_{2}, \\
& \left(1-\alpha_{3}\right)\left(\mathbf{D}_{\alpha_{3}}\left(Q_{P, W_{s}} \| P R_{P, W_{s}}\right)\right. \\
& \left.+\varepsilon-\mathbf{I}_{P, W_{s}}-\beta_{1}-\beta_{2}-\delta\right) \\
& \left.\left.\inf _{s \in \mathcal{S}} \mathbf{I}_{P, W_{s}}-\varepsilon-\mathcal{R}-\delta\right)\right)
\end{aligned}
$$

Since the exponent in (41) is then negative for sufficiently large $n$, we can combine it with (37), (38) and (39) to obtain (18).

\section{Cost Constraint in Compound Channel Coding and Re- solvability}

In this section, we prove Corollaries 1 and 3 which essentially state that the conclusions of Theorems 13 and 5 are also true for the case of cost-constrained codebooks. The approach used is similar to the one in [42, Section 3.3], but we include the adapted derivations in full here for the sake of selfcontainedness. We begin with a series of preliminary lemmas and conclude the section with the proofs of the corollaries.

Lemma 6. Let $\left(U_{k}\right)_{k \geq 1}$ be a sequence of independent and identically distributed random variables such that the moment generating function $\varphi(\lambda):=\mathbb{E} \exp \left(\lambda U_{1}\right)$ exists on an interval containing 0 in its interior. Let $C>\mathbb{E} U_{1}$. Then there exists $\gamma>0$ such that

$$
\mathbb{P}\left(\sum_{k=1}^{n} U_{k}>n C\right) \leq \exp (-n \gamma) .
$$

Proof. We can without loss of generality assume that $C=0$ and $\mathbb{E}\left(U_{1}\right)<0$, because otherwise we could consider the random variables $\left(U_{k}-C\right)_{k \geq 1}$ instead.

Clearly, $\varphi(0)=1$ and $\varphi^{\prime}(0)=\mathbb{E}\left(U_{1}\right)<0$, so we can find some $\lambda>0$ sufficiently small such that $\varphi(\lambda)<1$. With this choice of $\lambda$, we can apply Markov's inequality and get

$$
\begin{aligned}
\mathbb{P}\left(\sum_{k=1}^{n} U_{k}>0\right) & =\mathbb{P}\left(\exp \left(\lambda \sum_{k=1}^{n} U_{k}\right)>1\right) \\
& \leq \mathbb{E}\left(\exp \left(\lambda \sum_{k=1}^{n} U_{k}\right)\right) \\
& =\varphi(\lambda)^{n}
\end{aligned}
$$

so the lemma follows by choosing $\gamma:=-\log \varphi(\lambda)$.

Lemma 7. Let $\mathfrak{N}$ be a Bernoulli random variable with $\exp (n \mathcal{R})$ trials and success probability $p \leq \exp \left(-n \beta_{1}\right)$ where $\beta_{1}<\mathcal{R} / 2$. Then there are $\gamma_{1}, \gamma_{2}>0$ such that for sufficiently large $n$,

$$
\mathbb{P}\left(\mathfrak{N}>\exp \left(n\left(\mathcal{R}-\gamma_{1}\right)\right)\right) \leq \exp \left(-\exp \left(n \gamma_{2}\right)\right) .
$$


Proof. We choose $\gamma_{1}, \gamma_{2}$ and $\beta_{2}$ such that $0<\gamma_{1}<\beta_{1}<$ $\beta_{2}<\mathcal{R} / 2$ and $\gamma_{2}<\mathcal{R}-2 \beta_{2}$. Then

$$
\begin{aligned}
& \mathbb{P}\left(\mathfrak{N}>\exp \left(n\left(\mathcal{R}-\gamma_{1}\right)\right)\right) \\
= & \mathbb{P}\left(\mathfrak{N}>p \exp (n \mathcal{R})+\left(\exp \left(-n \gamma_{1}\right)-p\right) \exp (n \mathcal{R})\right) \\
\leq & \mathbb{P}\left(\mathfrak{N}>\mathbb{E} \mathfrak{N}+\left(\exp \left(-n \gamma_{1}\right)-\exp \left(-n \beta_{1}\right)\right) \exp (n \mathcal{R})\right) \\
\leq & \mathbb{P}\left(\mathfrak{N}>\mathbb{E} \mathfrak{N}+\exp \left(-n \beta_{2}\right) \exp (n \mathcal{R})\right) \\
\leq & \exp \left(-2 \frac{\left(\exp \left(-n \beta_{2}\right)\right)^{2}(\exp (n \mathcal{R}))^{2}}{\exp (n \mathcal{R})}\right) \\
= & \exp \left(-2 \exp \left(n\left(\mathcal{R}-2 \beta_{2}\right)\right)\right) \\
\leq & \exp \left(-\exp \left(n \gamma_{2}\right)\right)
\end{aligned}
$$

where (44) follows by the Chernoff-Hoeffding bound as stated for instance in [43, Theorem 1.1, eq. (1.6)].

Lemma 8. Let $P$ be a probability distribution on $\mathcal{X}$. Assume moreover that $c(X)$ has a moment generating function defined on an interval containing 0 in its interior and that $C>\mathbb{E}_{P} c(X)$. Denote the number of bad codewords in $\mathcal{C}$ with

$$
\mathfrak{N}:=\sum_{m=1}^{\exp (n \mathcal{R})} \mathbf{1}_{\sum_{j=1}^{n} c(\mathcal{C}(m)(j))>n C} .
$$

Then there are $\gamma_{1}, \gamma_{2}>0$ such that

$$
\mathbb{P}_{\mathcal{C}}\left(\mathfrak{N}>\exp \left(n\left(\mathcal{R}-\gamma_{1}\right)\right)\right) \leq \exp \left(-\exp \left(n \gamma_{2}\right)\right) .
$$

Proof. Since the codeword components are independently and identically distributed, we can apply Lemma 6 and obtain an arbitrarily small $\beta_{1}>0$ such that for all $m$,

$$
p:=\mathbb{P}_{\mathcal{C}}\left(\sum_{j=1}^{n} c(\mathcal{C}(m)(j))>n C\right) \leq \exp \left(-n \beta_{1}\right) .
$$

So since the codewords are independent, $\mathfrak{N}$ is a Bernoulli variable with $\exp (n \mathcal{R})$ trials and success probability $p$, and an application of Lemma 7 proves the conclusion.

Proof of Corollary $\square$ Assume throughout the proof that $n$ is sufficiently large. By Lemma 8 , we have $\hat{\gamma_{1}}, \hat{\gamma_{2}} \in(0, \infty)$ with

$$
\mathbb{P}_{\mathcal{C}}(\hat{\mathcal{E}}) \leq \exp \left(-\exp \left(n \hat{\gamma_{2}}\right)\right),
$$

where

$$
\hat{\mathcal{E}}:=\left\{\mathbb{P}_{\mathcal{M}}\left(\mathcal{C}(\mathcal{M}) \neq \mathcal{C}_{c, C}(\mathcal{M})\right)>\exp \left(-n \hat{\gamma_{1}}\right)\right\} .
$$

We denote the error of $\mathcal{C}$ with $\epsilon_{\mathcal{C}}$ and the error of $\mathcal{C}_{c, C}$ with $\epsilon_{\mathcal{C}_{c, C}}$. By Theorem 3 and Markov's inequality, we have, for some $\hat{\gamma} \in(0, \infty)$ given by the theorem and with choices $\tilde{\gamma_{1}} \in$ $\left(0, \min \left(\hat{\gamma}, \hat{\gamma_{1}}\right)\right), \tilde{\gamma_{2}} \in\left(0, \hat{\gamma}-\tilde{\gamma_{1}}\right)$,

$$
\begin{aligned}
\mathbb{P}_{\mathcal{C}}\left(\epsilon_{\mathcal{C}} \geq \exp \left(-n \tilde{\gamma_{1}}\right)\right) & \leq \mathbb{E}_{\mathcal{C}} \epsilon_{\mathcal{C}} \exp \left(n \tilde{\gamma_{1}}\right) \\
& \leq \exp \left(-n\left(\hat{\gamma}-\tilde{\gamma_{1}}\right)\right) \\
& \leq \exp \left(-n \tilde{\gamma_{2}}\right) .
\end{aligned}
$$

Conditioned on the complement of $\hat{\mathcal{E}}$, we have

$$
\begin{aligned}
\epsilon_{\mathcal{C}_{c, C}} & \stackrel{(a)}{=} \sup _{s \in \mathcal{S}} \mathbb{E}_{\mathcal{M}}\left(\mathbb{P}_{s}\left(\mathcal{M} \neq d\left(Y^{n}\right) \mid X^{n}=\mathcal{C}_{c, C}(\mathcal{M})\right)\right) \\
= & \sup _{s \in \mathcal{S}} \sum_{m=1}^{\exp (n \mathcal{R})} \exp (-n \mathcal{R}) \mathbb{P}_{s}\left(m \neq d\left(Y^{n}\right) \mid X^{n}=\mathcal{C}_{c, C}(m)\right) \\
& \stackrel{(b)}{\leq} \sup _{s \in \mathcal{S}} \sum_{\substack{m=1 \\
\mathcal{C}_{c, C}(m)=\mathcal{C}(m)}}^{\exp (n \mathcal{R})} \exp (-n \mathcal{R}) \mathbb{P}_{s}\left(m \neq d\left(Y^{n}\right) \mid X^{n}=\mathcal{C}_{c, C}(m)\right) \\
& +\sum_{\substack{m=1 \\
\exp (n \mathcal{R})}} \exp (-n \mathcal{R}) \\
& \stackrel{(a)}{\leq} \epsilon_{\mathcal{C}}+\exp \left(-n \hat{\gamma_{1}}\right),
\end{aligned}
$$

where the steps marked with (a) are by the definition of compound coding error, and (b) is by upper bounding some of the probabilities in the sum with 1 . We can now choose $\gamma_{1} \in\left(0, \tilde{\gamma_{1}}\right)$ and obtain

$$
\begin{aligned}
& \quad \mathbb{P}_{\mathcal{C}}\left(\epsilon_{\mathcal{C}_{c, C}} \geq \exp \left(-n \gamma_{1}\right)\right) \\
& \stackrel{(a)}{\leq} \mathbb{P}_{\mathcal{C}}\left(\epsilon_{\mathcal{C}_{c, C}} \geq \exp \left(-n \gamma_{1}\right) \mid \neg \hat{\mathcal{E}}\right)+\mathbb{P}_{\mathcal{C}}(\hat{\mathcal{E}}) \\
& \stackrel{\text { 50] }}{\leq} \mathbb{P}_{\mathcal{C}}\left(\epsilon_{\mathcal{C}}+\exp \left(-n \hat{\gamma_{1}}\right) \geq \exp \left(-n \gamma_{1}\right) \mid \neg \hat{\mathcal{E}}\right)+\mathbb{P}_{\mathcal{C}}(\hat{\mathcal{E}}) \\
& \stackrel{(a)}{\leq} \frac{\mathbb{P}_{\mathcal{C}}\left(\epsilon_{\mathcal{C}} \geq \exp \left(-n \gamma_{1}\right)-\exp \left(-n \hat{\gamma_{1}}\right)\right)}{1-\mathbb{P}_{\mathcal{C}}(\hat{\mathcal{E}})}+\mathbb{P}_{\mathcal{C}}(\hat{\mathcal{E}}) \\
& \stackrel{(b)}{\leq} \frac{\mathbb{P}_{\mathcal{C}}\left(\epsilon_{\mathcal{C}} \geq \exp \left(-n \tilde{\gamma_{1}}\right)\right)}{1-\mathbb{P}_{\mathcal{C}}(\hat{\mathcal{E}})}+\mathbb{P}_{\mathcal{C}}(\hat{\mathcal{E}}) \\
& \stackrel{\text { (48), ,49 }}{\leq} \frac{\exp \left(-n \tilde{\gamma_{2}}\right)}{1-\exp \left(-\exp \left(n \hat{\gamma_{2}}\right)\right)}+\exp \left(-\exp \left(n \hat{\gamma_{2}}\right)\right) \\
& \stackrel{(c)}{\leq} \exp \left(-n \gamma_{2}\right),
\end{aligned}
$$

where the steps marked with (a) are by the law of total probability, step (b) is by the choices of $\gamma_{1}, \tilde{\gamma_{1}}$, and step (c) is valid for any choice of $\gamma_{2} \in\left(0, \tilde{\gamma_{2}}\right)$.

Proof of Corollary 3 By Lemma 8 , we pick $\hat{\gamma_{1}}, \hat{\gamma_{2}}$ satisfying (47) and by Theorem [5, we pick $\tilde{\gamma_{1}}, \tilde{\gamma_{2}}$ satisfying (26).

We use the observation that $\mathfrak{N} \leq \exp \left(\left(\mathcal{R}-\hat{\gamma_{1}}\right) n\right)$ implies

$$
\left\|R_{\mathcal{C}_{c, C}}-R_{\mathcal{C}}\right\|_{\mathrm{TV}} \leq \frac{\mathfrak{N}}{\exp (n \mathcal{R})} \leq \exp \left(-\hat{\gamma_{1}} n\right)
$$

and observe that, as long as $\gamma_{1}<\hat{\gamma_{1}}, \tilde{\gamma_{1}}$ and $\gamma_{2}<\hat{\gamma_{2}}, \tilde{\gamma_{2}}$ and $n$ is sufficiently large,

$$
\begin{aligned}
& \quad \mathbb{P}_{\mathcal{C}_{c, C}}\left(\left\|R_{\mathcal{C}_{c, C}}-Q_{P, W}^{n}\right\|_{\mathrm{TV}}>\exp \left(-\gamma_{1} n\right)\right) \\
& \stackrel{(a)}{\leq} \mathbb{P}_{\mathcal{C}}\left(\left\|R_{\mathcal{C}_{c, C}}-R_{\mathcal{C}}\right\|_{\mathrm{TV}}+\left\|R_{\mathcal{C}}-Q_{P, W}^{n}\right\|_{\mathrm{TV}}>\exp \left(-\gamma_{1} n\right)\right) \\
& \stackrel{(b)}{\leq} \mathbb{P}_{\mathcal{C}}\left(\left\|R_{\mathcal{C}_{c, C}}-R_{\mathcal{C}}\right\|_{\mathrm{TV}}>\exp \left(-\hat{\gamma_{1}} n\right)\right) \\
& \quad+\mathbb{P}_{\mathcal{C}}\left(\left\|R_{\mathcal{C}}-Q_{P, W}^{n}\right\|_{\mathrm{TV}}>\exp \left(-\tilde{\gamma}_{1} n\right)\right) \\
& \stackrel{(c)}{<} \exp \left(-\exp \left(\hat{\gamma_{2}} n\right)\right)+\exp \left(-\exp \left(\tilde{\gamma_{2}} n\right)\right) \\
& \stackrel{(d)}{\leq} \exp \left(-\exp \left(\gamma_{2} n\right)\right),
\end{aligned}
$$


where (a) is by the triangle inequality, (b) is by the union bound and the choice of $\gamma_{1}$, (c) is due to (47), (51) and 26), and (d) is by the choice of $\gamma_{2}$.

\section{CONCLUSION}

In this work, we have introduced a framework for distributed function approximation schemes with jamming. We have shown how well-known information theoretic tools can be used to improve security by means of a jammer whose signal is stronger at the legitimate receiver than it is at the eavesdropper. In the process, we have proved a compound channel coding result which is a generalization of similar results from the literature. For the special case of an AWGN channel, we have proven MSE guarantees both for the eavesdropper's and the legitimate receiver's reconstruction of the objective function. For the case of fast-fading sub-Gaussian channels, we have presented a distributed function approximation scheme with theoretical guarantees for the tail probabilities of the absolute reconstruction error.

This work is intended as an initial step towards providing security against eavesdropping for OTA computation schemes. We have given theoretical analysis which shows that a large class of such schemes can be made more secure, but a gap between this theoretical work and its implementation for the envisioned practical applications remains. In particular, we are interested in the following questions for future research:

- Can the secrecy guarantees in this work be achieved with structured codes which allow for practically feasible encoding and decoding?

- Can the secrecy guarantees be strengthened to full semantic security?

\section{ACKNOWLEDGMENT}

The authors wish to thank the anonymous reviewer whose comments have greatly helped to improve the overall quality and in particular the readability and structure of this paper.

\section{REFERENCES}

[1] M. Frey, I. Bjelakovic, and S. Stanczak, "Towards secure over-the-air computation," in 2021 IEEE International Symposium on Information Theory (ISIT). IEEE, 2021.

[2] M. M. Amiri and D. Gündüz, "Machine learning at the wireless edge: Distributed stochastic gradient descent over-the-air," IEEE Transactions on Signal Processing, vol. 68, pp. 2155-2169, 2020.

[3] K. Ralinovski, M. Goldenbaum, and S. Stańczak, "Energy-efficient classification for anomaly detection: The wireless channel as a helper," in 2016 IEEE International Conference on Communications (ICC), 2016, pp. 1-6.

[4] I. Bjelaković, M. Frey, and S. Stańczak, "Distributed approximation of functions over fast fading channels with applications to distributed learning and the max-consensus problem," in 57th Annual Allerton Conference. IEEE, 2019.

[5] M. Frey, I. Bjelaković, and S. Stańczak, "Over-the-air computation in correlated channels," in 2020 IEEE Information Theory Workshop (ITW). IEEE, 2021, pp. 1-5.

[6] M. Frey, I. Bjelaković, and S. Stańczak, "Over-the-air computation in correlated channels," IEEE Transactions on Signal Processing, vol. 69, pp. 5739-5755, 2021.

[7] W. Liu, X. Zang, Y. Li, and B. Vucetic, "Over-the-air computation systems: Optimization, analysis and scaling laws," IEEE Transactions on Wireless Communications, vol. 19, pp. 5488-5502, 2020.

[8] W. L. Root and P. P. Varaiya, "Capacity of classes of gaussian channels," SIAM J. Appl. Math., vol. 16, no. 6, pp. 1350-1393, 1968.
[9] B. Nazer and M. Gastpar, "Computation over multiple-access channels," IEEE Trans. Inf. Theory, vol. 53, no. 10, pp. 3498-3516, 2007.

[10] M. Goldenbaum and S. Stanczak, "Robust analog function computation via wireless multiple-access channels," IEEE Trans. Commun., vol. 61, no. 9, pp. 3863-3877, 2013.

[11] R. L. Dobrushin, "Optimum information transmission through a channel with unknown parameters," Radio Eng. Electron, vol. 4, no. 12, pp. 1-8, 1959.

[12] D. Blackwell, L. Breiman, and A. J. Thomasian, "The capacity of a class of channels," Ann. Math. Stat., vol. 30, no. 4, pp. 1229-1241, 1959.

[13] J. Wolfowitz, "Simultaneous channels," Arch. Ration. Mech. Anal., vol. 4, no. 1, pp. 371-386, 1959.

[14] H. Kesten, "Some remarks on the capacity of compound channels in the semicontinuous case," Inf. Control, vol. 4, no. 2-3, pp. 169-184, 1961.

[15] K. Yoshihara, "Coding theorems for the compound semi-continuous memoryless channels," in Kodai Mathematical Seminar Reports, vol. 17, no. 1. Department of Mathematics, Tokyo Institute of Technology, 1965, pp. 30-43.

[16] R. Ahlswede, "Certain results in coding theory for compound channels," in Proceedings of the Colloquium on Information Theory, 1967.

[17] A. Wyner, "The common information of two dependent random variables," Trans. Inf. Theory, vol. 21, no. 2, pp. 163-179, 1975.

[18] T. S. Han and S. Verdú, "Approximation theory of output statistics," Trans. Inf. Theory, vol. 39, no. 3, pp. 752-772, 1993.

[19] I. Csiszár, "Almost independence and secrecy capacity," Problems Inform. Transmission, vol. 32, no. 1, pp. 40-47, 1996.

[20] I. Devetak, "The private classical capacity and quantum capacity of a quantum channel," Trans. Inf. Theory, vol. 51, no. 1, pp. 44-55, 2005.

[21] M. Hayashi and R. Matsumoto, "Secure multiplex coding with dependent and non-uniform multiple messages," IEEE Transactions on Information Theory, vol. 62, no. 5, pp. 2355-2409, 2016.

[22] P. Cuff, "Soft covering with high probability," in 2016 IEEE International Symposium on Information Theory (ISIT). IEEE, 2016, pp. 2963 2967.

[23] M. Frey, I. Bjelakovic, and S. Stanczak, "Resolvability on continuous alphabets," in 2018 IEEE International Symposium on Information Theory (ISIT). IEEE, 2018, pp. 2037-2041.

[24] M. Hayashi, "General nonasymptotic and asymptotic formulas in channel resolvability and identification capacity and their application to the wiretap channel," IEEE Transactions on Information Theory, vol. 52, no. 4, pp. 1562-1575, 2006.

[25] I. Csiszar and J. Körner, Information theory: coding theorems for discrete memoryless systems. Cambridge: Cambridge University Press, 2011.

[26] M. Bellare, S. Tessaro, and A. Vardy, "Semantic security for the wiretap channel," in Advances in Cryptology-CRYPTO 2012. Springer, 2012, pp. 294-311.

[27] M. R. Bloch and J. N. Laneman, "Strong secrecy from channel resolvability," Trans. Inf. Theory, vol. 59, no. 12, pp. 8077-8098, 2013.

[28] R. Negi and S. Goel, "Secret communication using artificial noise," in IEEE vehicular technology conference, vol. 62, no. 3. IEEE, 2005, p. 1906.

[29] J. P. Vilela, M. Bloch, J. Barros, and S. W. McLaughlin, "Friendly jamming for wireless secrecy," in 2010 IEEE International Conference on Communications. IEEE, 2010, pp. 1-6.

[30] - "Wireless secrecy regions with friendly jamming," IEEE Transactions on Information Forensics and Security, vol. 6, no. 2, pp. 256-266, 2011.

[31] I. Stanojev and A. Yener, "Improving secrecy rate via spectrum leasing for friendly jamming," IEEE Transactions on Wireless Communications, vol. 12, no. 1, pp. 134-145, 2012.

[32] C. E. Shannon, "Communication theory of secrecy systems," Bell Labs Technical Journal, vol. 28, no. 4, pp. 656-715, 1949.

[33] A. D. Wyner, "The wire-tap channel," Bell Labs Technical Journal, vol. 54, no. 8, pp. 1355-1387, 1975.

[34] U. M. Maurer, "The strong secret key rate of discrete random triples," in Communications and Cryptography: Two Sides of One Tapestry, R. E. Blahut, D. J. Costello, U. Maurer, and T. Mittelholzer, Eds. Boston, MA: Springer US, 1994, pp. 271-285.

[35] J. Hou and G. Kramer, "Effective secrecy: Reliability, confusion and stealth," in 2014 IEEE International Symposium on Information Theory. IEEE, 2014, pp. 601-605.

[36] Z. Utkovski, P. Agostini, M. Frey, I. Bjelakovic, and S. Stanczak, "Learning radio maps for physical-layer security in the radio access," in 2019 IEEE 20th International Workshop on Signal Processing Advances in Wireless Communications (SPAWC). IEEE, 2019. 
[37] D. Klinc, J. Ha, S. W. McLaughlin, J. Barros, and B.-J. Kwak, "Ldpc codes for the gaussian wiretap channel," IEEE Transactions on Information Forensics and Security, vol. 6, no. 3, pp. 532-540, 2011.

[38] T. van Erven and P. Harremos, "Rényi divergence and Kullback-Leibler divergence," IEEE Trans. Inf. Theory, vol. 60, no. 7, pp. 3797-3820, 2014.

[39] M. Gil, "On Rényi divergence measures for continuous alphabet sources," Master's thesis, Queen's University Kingston, Ontario, Canada, 2011.

[40] E. Jaynes, Probability Theory: The Logic of Science. Cambridge: Cambridge University Press, 2003.

[41] N. L. Johnson, S. Kotz, and N. Balakrishnan, Continuous Univariate Distributions, 2nd ed., ser. Wiley Series in Probability and Mathematical Statistics. New York, Chichester, Brisbane, Toronto, Singapore: Wiley, 1994, vol. 1.

[42] A. El Gamal and Y.-H. Kim, Network information theory. Cambridge: Cambridge university press, 2011.

[43] D. P. Dubhashi and A. Panconesi, Concentration of Measure for the Analysis of Randomized Algorithms. Cambridge University Press, 2009. 